\title{
An experimental investigation into the micromechanics of non-active clays
}

\author{
M. PEDROTTI* and A. TARANTINO $\dagger$
}

\begin{abstract}
The paper presents an experimental investigation into the micro-mechanisms controlling the behaviour of non-active clays. Clay microstructural behaviour was investigated by way of mercury intrusion porosimetry (MIP) accompanied by scanning electron microscope images. To gain insight into the mechanisms underlying reversible and non-reversible compression, samples for MIP testing were taken along both normal compression and unloading-reloading lines. To investigate the nature of interparticle forces, the response of clay samples prepared with deionised water (characterised by acidic $\mathrm{pH}$ ) was compared with clay samples prepared with alkaline water. A high $\mathrm{pH}$ 'deactivates' the edge-to-face contacts that are indeed active in the clay prepared with deionised (acidic) water. The pore size distribution data clearly highlighted that the smaller pores are associated with particles in non-contact configuration, that is only interacting by way of the overlap of the repulsive electrical field generated by the negatively charged faces. On the other hand, larger pores are associated with contact configuration, generated by the attraction between the positively charged edge and the negatively charged face of the clay particle. The pore size distribution data also made it possible to infer that reversible behaviour is mainly associated with the reversible overlap of the repulsive electrical field in contact configuration, whereas the plastic response appears to be associated, at the micro-scale, with the loss of edge-to-face contacts. Finally, an embryonic one-dimensional discrete-element method (DEM) model was developed to show the potential of the micromechanical conceptual model to be implemented into a DEM model.
\end{abstract}

KEYWORDS: chemical properties; clays; discrete-element modelling; fabric/structure of soils; microscopy; particle-scale behaviour

\section{INTRODUCTION}

The mechanical response of geomaterials is controlled by the interactions between particles. An understanding of such interactions is a critical step in soil mechanics as it allows researchers to interpret experimental data, to understand fundamental mechanisms of behaviour, to inform 'continuum' constitutive models and to develop discrete-element method (DEM) approaches.

Significant advances have been made in the micromechanics of granular materials. The interplay between particle-scale interactions and the overall observed geomaterial response including small strain stiffness, shear strain localisation, friction, yielding and crushing has been investigated experimentally by way of X-ray computed tomography (Oda \& Kazama, 1998; Oda et al., 1998; Andò et al., 2012a, 2012b; Zhao et al., 2015) and explored using analytical or DEM modelling (O'Sullivan, 2011).

Research in micromechanics of clayey geomaterials, however, is lagging behind owing to the difficulty of investigating particle-to-particle interaction at the microscale. Even very basic responses observed in oedometer compression, that is irreversible deformation along the virgin compression line and 'reversible' deformation along the

Manuscript received 21 September 2016; revised manuscript accepted 21 September 2017

Discussion on this paper is welcomed by the editor.

* Department of Civil and Environmental Engineering, University of Strathclyde, Glasgow, UK (Orcid:0000-0002-2495-1711).

$\dagger$ Department of Civil and Environmental Engineering, University of Strathclyde, Glasgow, UK. unloading-reloading line, are not elucidated by basic microscale mechanisms.

Characterisation of micro-mechanisms involves the configuration of elements (particles) and the forces controlling the kinematics of such elements. Particle configuration in clays upon loading has rarely been discussed explicitly, although significant aspects of the pore space and particle configuration have been addressed by mercury intrusion porosimetry (MIP) and scanning electron microscope (SEM) testing, respectively (Delage \& Lefebvre, 1984; Delage, 2010; Hattab et al., 2010). However, researchers have speculated about the nature of inter-particle forces. These can be divided into two different categories according to Sridharan \& Venkatappa Rao (1973): (a) contact forces, which are transferred from one particle to another through a contact surface and $(b)$ non-contact forces such as Coulombian repulsion/attraction forces associated with the diffuse double layer and Van der Waals attraction forces. Contact forces are often referred to as mechanical and non-contact forces as electro-chemical.

When describing the dominant forces in clayey material, a distinction should be made between active clays (characterised by a 2:1 mineralogical structure) and non-active clays (characterised by a 1:1 mineralogical structure). For the case of active clays, it is widely accepted that dominant forces are electro-chemical (Bolt, 1956; Sridharan \& Venkatappa Rao, 1973; Madsen \& Müller-Vonmoos, 1989; Di Maio \& Fenelli, 1994; Gajo \& Loret, 2007). Such an assumption is supported by the fair agreement between experimental data and qualitative prediction inferred from theoretical models describing the interaction between two particles (diffuse double layer). For example, Di Maio (1996) showed that experimental shear strength results were consistent with the hypothesis that the thickness of the diffuse double layer 
changes in response to changes in pore-water salt concentration.

On the other hand, the nature of interaction forces in non-active clays is quite unclear. Many authors, such as Sloane \& Kell (1966), Sridharan \& Venkatappa Rao (1973) and Wahid et al. (2011b), have suggested that particle contact exists in non-active clays and body friction develops between particles (i.e. dominant inter-particle interaction would be mechanical).

Such a conclusion is consistent with one of the key assumptions behind the 'original' Cam Clay model (Roscoe et al., 1963; Roscoe \& Burland, 1968). Plastic deformations are ruled by friction mechanisms; that is, energy is dissipated by particle friction when yielding occurs. At the same time, it has been shown that shear strength and volume change behaviour are generally affected by changes in the chemical composition of the pore fluid, implicitly suggesting that electro-chemical forces also control inter-particle interactions to some extent (Sridharan \& Venkatappa Rao, 1973; Chen et al., 2000; Wang \& Siu, 2006; Sachan \& Penumadu, 2007; Wahid et al., 2011b).

The three factors characterising the pore-fluid chemistry are the electrolyte concentration, the $\mathrm{pH}$ and the dielectric permittivity. According to Wang \& Siu (2006) and Wahid et al. (2011a) mechanical behaviour of kaolinite (1:1 mineralogical structure) is not affected by pore fluid electrolyte concentration. In contrast, Chen et al. (2000) noted that, even though the change in compressibility upon electrolyte adjunction was noticeable only for overburden stress lower than $300 \mathrm{kPa}$, this was coherent with the double layer interaction.

The pore fluid $\mathrm{pH}$, however, seems to influence kaolin mechanical behaviour significantly by controlling edge particle charges (Wang \& Siu, 2006; Sachan \& Penumadu, 2007; Wahid et al., 2011a). Moreover, non-recoverable displacements after acid or alkaline exposure may be related to particle dissolution (Wahid et al., 2011a).

Concerning the effects of dielectric permittivity, mechanical effects appear to correlate slightly with the dielectric permittivity of the pore fluid according to Wahid et al. (2011a). On the other hand, Sridharan \& Venkatappa Rao (1973) showed that equilibrium void ratio at a particular external pressure decreases monotonically as the dielectric permittivity of the pore fluid increases and Chen et al. (2000) concluded that compressibility varies with a non-monotonic trend with the dielectric permittivity and with a trend comparable with the Van der Waals force dependency on dielectric permittivity.

In conclusion, the questions remain open about the nature of inter-particle forces in non-active clays, the interplay between inter-particle forces and particle configuration, and to what extent mechanical and electrochemical forces control specific aspects of mechanical behaviour (e.g. plastic and elastic response).

The aim of this paper is to investigate the micromechanics of non-active clays in an attempt to elucidate some of the above questions. The approach pursued in this work is to monitor the evolution of the pore size distribution (PSD) along non-reversible and reversible volume changes while purposely modulating specific electrochemical forces to explore their micromechanical effects.

\section{THE NATURE OF PARTICLE INTERACTION IN KAOLIN CLAY}

The kaolin mineral structure is created by alternating silica tetrahedral sheets and alumina octahedral sheets (two sheets per unit cell). Mineral particles of kaolinite consist in unit cell stacked in the c-direction (direction perpendicular to the clay layers). In general, unit cells may be stacked closely together (as in non-active clays) or water layer may intervene (as in active clays). In kaolin, the bonding between successive layers (both Van der Waals and hydrogen bonds) is sufficiently strong to prevent interlayer swelling in the presence of water (kaolin is indeed considered a non-active clay). A single particle is therefore considered to be a non-deformable elementary unit.

Two dominant forces can be considered to control the interaction between clay particles, the Coulombian forces arising from the permanent charge on face and edge of the clay particle and Van der Waals attraction forces resulting from temporary dipoles. Van der Waals forces are always attractive whereas Coulombian forces may be attractive or repulsive depending on the sign of the opposing charges.

The charge of the particle face (surface charge on the basal planes) results from isomorphic substitutions and is always considered to be negative (Van Olphen, 1977), although its magnitude appears to be $\mathrm{pH}$ dependent (Gupta \& Miller, 2010). The charge of the particle edge results from the protonation or deprotonation of hydroxyl groups of edges and, unlike the face, the sign of the edge charge is $\mathrm{pH}$ dependent (Schofield \& Samson, 1954; Rand \& Melton, 1977; Van Olphen, 1977; Braggs et al., 1994; Ganor et al., 1995; Brady \& Weil, 1996; Huertas et al., 1998; Kretzschmar et al., 1998; Carty, 1999; Mitchell \& Soga, 2005; Wang \& Siu, 2006; Gupta et al., 2011).

Titration curves generally show the point of zero charge of the edges (PZC) for kaolin to be about pH 5.5 (Sposito, 1984; Carroll \& Walther, 1990; Huertas et al., 1998). Accordingly, the edges of kaolinite particles become positively charged when kaolin suspension is acidic (Fig. 1(a)) and negatively charged when kaolin suspension is alkaline (Fig. 1(b)). Neutralisation of positive edges in alkaline solutions is associated with deprotonation of the edges, where potential sites for hydroxyl anions $\left(\mathrm{OH}^{-}\right)$are present.

The interaction between clay particles, which results from the balance between Coulombian interactions (either attractive or repulsive) and Van der Waals attraction, has been investigated extensively for the case of clay particles in suspension. The commonly accepted theory used to interpret interactions observed experimentally is referred to as the DLVO theory, named after Boris Derjaguin and Lev Landau, Evert Verwey and Theodoor Overbeek (Derjaguin \& Landau, 1941; Verwey et al., 1948).

Coulombian interaction between particle surfaces depends on the electric field generated by the particle charges. The interaction can be attractive if the surfaces are charged with opposite sign or repulsive if the sign of the charges is the same.

In general, Coulombian forces depend on the dielectric permittivity and electrolyte concentration of the pore fluid, and the inter-particle distance (Coulomb's law). In an electrolyte-free suspension, Van der Waals forces are considered to be relevant (compared to the Coulombian forces) only at distances smaller than 10-20 nm (Van Olphen, 1977; Mitchell \& Soga, 2005). Therefore, as the minimum pore size typically observed in soils usually appears to be at least one order of magnitude greater $(>200 \mathrm{~nm})$, particle distance can be mainly assumed to be controlled by the Coulombian interactions.

Particles are considered to interact in two different modes.

(a) Face-to-face interaction: Coulombian repulsion exists between the two negatively charged faces (Figs 1(a) and $1(\mathrm{~b}))$.

(b) Edge-to-face interaction: Coulombian attraction (Fig. 1(a)) exists between the negative clay faces and the positive edges (edges are positively charged as long as the solution is not alkaline and no dispersant is added) 


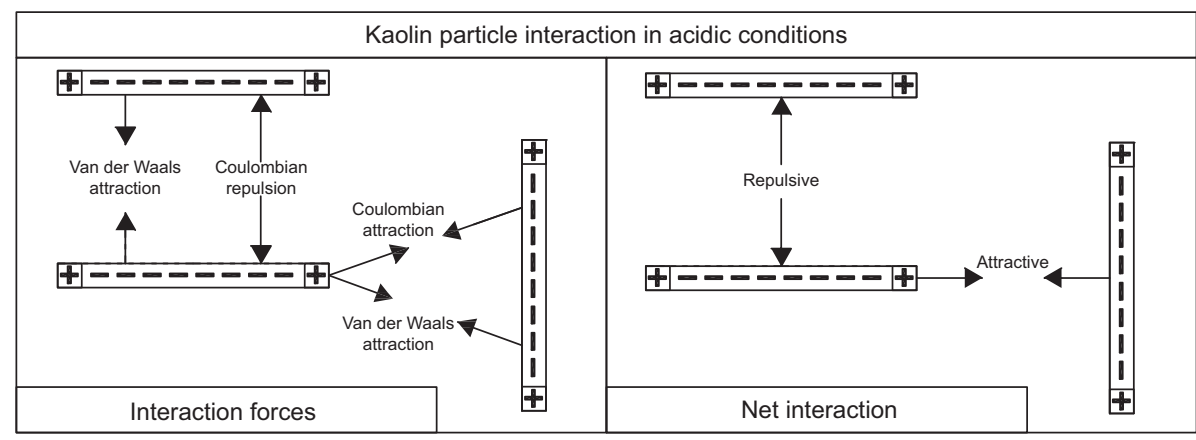

(a)

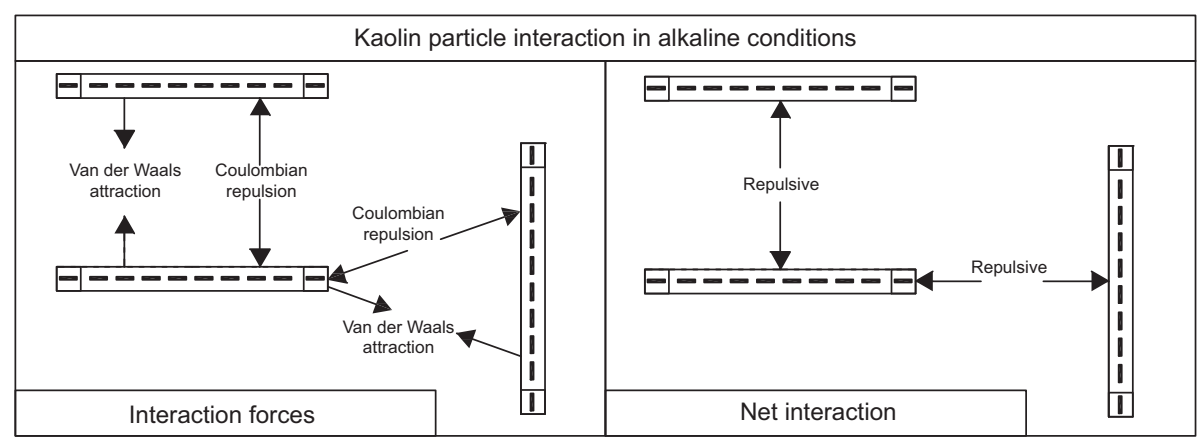

(b)

Fig. 1. Electrochemical interaction forces of kaolin particles in: (a) acidic water; (b) dispersant solution

for the case of acidic solutions or Coulombian repulsion between negatively charged faces and negatively charged edges (Fig. 1(b)) for the case of alkaline solutions.

Regardless of whether the interaction is attractive or repulsive, the intensity of the inter-particle Coulombian forces depends on surface charge and dielectric constant of the pore fluid.

For the case of two facing circular plates, Coulomb's force is inversely proportional to the particle distance and dielectric permittivity as shown in equation (1) (see Appendix)

$$
F=\frac{\pi a^{2}}{8 \varepsilon_{0} \varepsilon_{\mathrm{r}}} \rho^{2}\left(\frac{z}{\sqrt{a^{2}+z^{2}}}-1\right)
$$

where $a$ is the radius of the plates (m); $z$ is the plate distance (m); $\rho$ is the electrical charge distribution on the two plates $\left(\mathrm{C} / \mathrm{m}^{2}\right) ; \varepsilon_{0}(\mathrm{~F} / \mathrm{m})$ is the vacuum permittivity; and $\varepsilon_{\mathrm{r}}$ is the relative permittivity of the medium between the two plates. As a result, a change in pore-fluid chemistry gives rise to a change in inter-particle interactions and configuration. Fig. 2 shows the Coulomb's force for different pore fluid dielectric permittivities.

\section{MATERIALS AND SPECIMENS PREPARATION}

Speswhite kaolin with plastic limit $w_{\mathrm{P}}=0.32$ and liquid limit $w_{\mathrm{L}}=0.64$ was chosen for tests presented in this paper. The grain size distribution showed it to have $0 \cdot 20$ silt fraction and 0.80 clay fraction. Three different kinds of pore fluid were used: acidic water, alkaline water and acetone $(\geq 99 \cdot 9 \%)$.

Four different mixtures were prepared for this research work: dry powder, acidic kaolin mixture, alkaline kaolin mixture and kaolin mixed with acetone. Dry powder was prepared by keeping kaolin powder in the oven $\left(105^{\circ} \mathrm{C}\right)$ for at least $24 \mathrm{~h}$ and then cooling down in a dessicator. During the

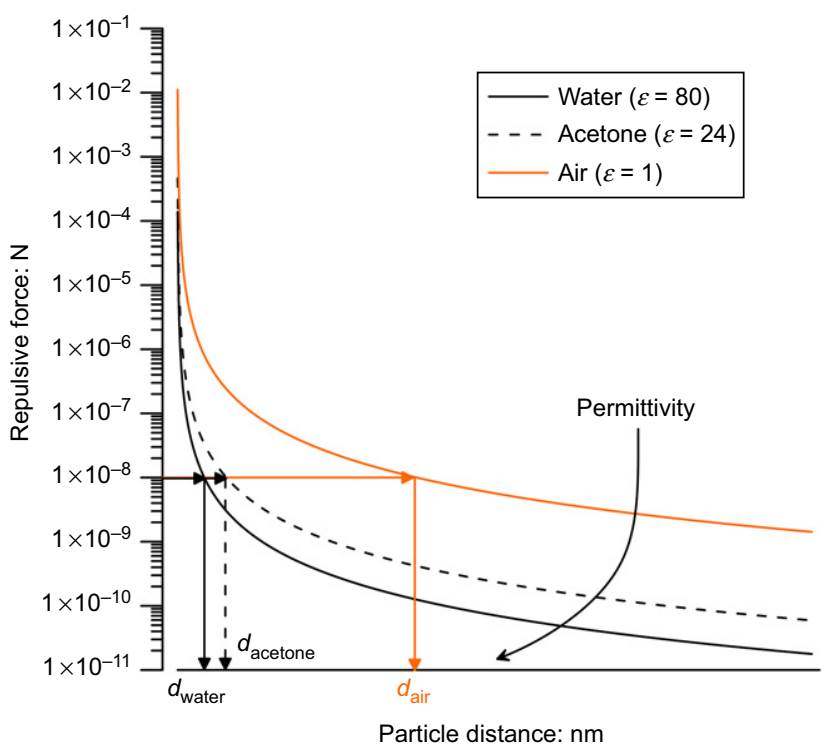

Fig. 2. Repulsive force for three different pore fluids: water, acetone and air

experiments, no particular attention was paid to prevention of capillary condensation because the hygroscopic water content was shown to be less than $0 \cdot 08$.

Kaolin samples were prepared under acidic $(\mathrm{pH}=4)$ or alkaline $(\mathrm{pH} \sim 9)$ conditions, that is with $\mathrm{pH}$ lower and higher than the PZC ( $\mathrm{pH}$ measured after mixing). The purpose of the acidic sample is to reproduce the 'ordinary' clay tested in the laboratory when prepared from slurry by mixing kaolinite powder with deionised water $(\mathrm{pH}$ of slurry prepared in this way is typically acidic). The purpose of the alkaline sample is to modify the charge of the particle edge from positive to negative values. This 'deactivates' the edge-to-face attraction between clay particles that occurs in acidic samples. 
Acidic water was prepared by adding a few drops of very dilute solution of hydrochloric acid ( $\mathrm{HCl}$ solution) to deionised water to obtain a $\mathrm{pH}$ equal to 4 after exposure to clay. The total concentration of hydrochloric salt $(\mathrm{HCl})$ at the end of the preparation was always less than $0.0001 \mathrm{M}$, which has been proved to be negligible in terms of electrolyte concentration (Pedrotti, 2016).

A solution of potassium hydroxide $(\mathrm{KOH})$ with a molar concentration smaller than $0 \cdot 01 \mathrm{M}$ was used to prepare alkaline water. Such an amount of electrolyte was sufficient to raise the $\mathrm{pH}$ of the mixture to values higher than 9 after exposure to clay, and was proven to be negligible in terms of electrolyte concentration (Pedrotti, 2016).

\section{EXPERIMENTAL PROCEDURES}

Two different tests were performed on the clay: a sedimentation test and one-dimensional (1D) mechanical compression.

Sedimentation tests were performed according to BS 1377-2:1990 (BSI, 1990). However, instead of using only a dispersant solution, the clay was either mixed with acidic or alkaline water.

The 1D mechanical compression tests were performed in an oedometer cell (dia. $75 \mathrm{~mm}$ ). In one series of tests, the oedometer ring was initially filled with oven-dried powder. After applying a vertical stress of about $1 \mathrm{kPa}$ (associated with the weight of the loading cap), the cell was either saturated with the required fluid (acidic water, alkaline water, or acetone) or left dry. Upon imbibition, time was allowed for the sample to fully consolidate $(c .2 \mathrm{~h})$. Afterwards, samples were compressed in steps to the target vertical stress. Unloading was performed in one single step. Samples were allowed to fully consolidate under any loading step. Consolidation upon unloading steps was either allowed fully or hindered, as explained later in the paper. As vertical displacement was monitored during the test, the void ratio could be back-calculated at any stage of the test from the final fluid content measured at the end of the test.

In a second series of tests, kaolin clay (acidic mixture) was reconstituted from slurry. The slurry was prepared with a water content $1.5 w_{\mathrm{L}}$ and then placed in the oedometer ring. The loading sequence was the same as discussed above.

Mercury intrusion porosimeter tests were carried out using a Poremaster-60 manufactured by Quantachrome Instruments. The equipment is designed to measure pore entrance diameters in the range from about $1000 \mu \mathrm{m}$ to $0.003 \mu \mathrm{m}$ corresponding to 1.5 to $420000 \mathrm{kPa}$ mercury intrusion pressure, respectively. For pressure up to $340 \mathrm{kPa}$ the machine operates on a station different from the one used for high pressures. Blank tests were performed for the two porosimeters to correct errors associated with system compressibility and temperature effects. Intrusion rate was selected in order to minimise the temperature increase in the system.

Microscope images were obtained by means of field emission scanning electron microscope (FE-SEM) (Hitachi SU-6600). The FE-SEM is equipped with energy dispersive spectroscopy (EDS), Oxford Inca 350 with $20 \mathrm{~mm}$ X-Max detector and wavelength dispersive spectroscopy (WDS), Oxford Inca Wave 700 microanalysis system with Energy.

All the water-saturated samples tested in the MIP and SEM (either acidic or alkaline) were dehydrated by a freeze-drying technique. The maximum time lag between the end of the sample preparation test and the freeze-drying equals 1-14 days (samples were stored in a sealed bag to preserve water content). Freeze-drying was performed by means of liquid nitrogen and isopentane, as described in Tarantino \& De Col (2008) and Pedrotti (2016). Samples containing acetone were instead dehydrated by placing them in the oven $\left(105^{\circ} \mathrm{C}\right)$ for $24 \mathrm{~h}$. Because of the low surface tension of the acetone sample, disturbance due to ovendrying was considered negligible. Air-saturated samples were not dehydrated.

To evaluate the sample disturbance associated with freeze-drying of water-saturated samples and oven-drying of acetone-saturated samples, the macroscopic void ratio and the void ratio extrapolated from the MIP test were compared.

For the SEM testing, specimens were gold coated before the electron scanning.

\section{EDGE-CHARGE EFFECT ON MACROSCOPIC BEHAVIOUR OF CONSOLIDATED CLAYS}

Figure 3(a) shows the results of sedimentation tests performed on acidic and alkaline specimens in terms of final void ratio. Results from the sedimentation tests from Wang \& Siu (2006) are also included in the figure.

The change in $\mathrm{pH}$ affects the response of the suspensions. The response changes markedly at a $\mathrm{pH}$ equal to the PZC value; such a change is usually associated with the change in the electrical charge of particle edges.

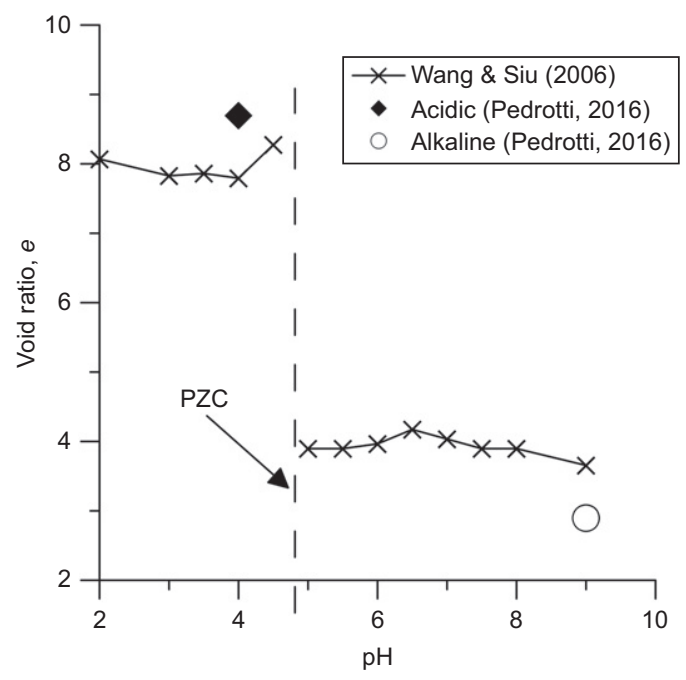

(a)

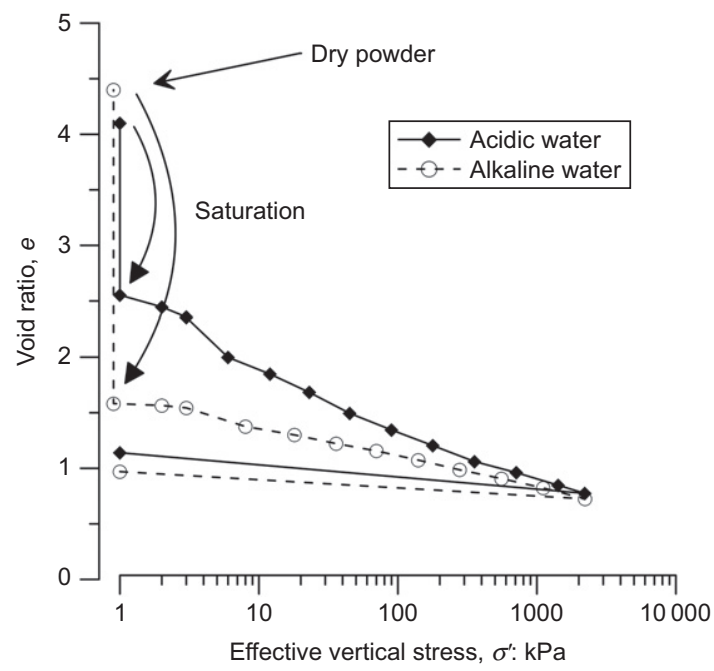

(b)

Fig. 3. Macroscopic behaviour for kaolin clay at different pH: (a) void ratio at the end of sedimentation tests performed on acidic and alkaline specimens; (b) 1D consolidation of acidic and alkaline samples 
For the case of $\mathrm{pH}<\mathrm{pH}_{\mathrm{PZC}}$ (active edge-to-face attractive interaction), particle configuration is assumed to be dominated by the edge-to-face interactions, which lead to a very open structure (Fig. 3(a)) (Van Olphen, 1977). For the case of $\mathrm{pH}>\mathrm{pH}_{\mathrm{PZC}}$ (de-activated edge-to-face attractive interaction), non-contact interactions between dispersed subparallel particles lead to a closer particle configuration (Fig. 3(a)) (Van Olphen, 1977).

Typical colloidal problems involve suspensions characterised by a clay fraction of less than $5 \%$ (weight of clay/weight of solution). In contrast, clays in soil mechanics problems are characterised by a clay fraction of greater than $100 \%$. The question then arises as to whether pore-water $\mathrm{pH}$ lower or higher than the PZC gives rise to the same step change in the mechanical response for the case of 'consolidated' clays.

Figure 3(b) shows the 1D consolidation of the sample saturated with acidic water and the sample saturated with dispersant solution. After cell saturation, a volumetric collapse was recorded. However, the two samples showed a different amount of collapse. The dry kaolin had an initial void ratio $e$ higher than 4 . After saturation with acidic water, the sample collapsed to a void ratio $e$ of about $2 \cdot 6$, whereas the sample prepared with the alkaline water collapsed to a void ratio of about $1 \cdot 5$. Upon loading, the sample saturated with acidic water was shown to be much more compressible, whereas the sample saturated with alkaline water showed an overconsolidated-like behaviour. At any given vertical stress, the sample prepared with acidic water always showed a larger void ratio than the sample prepared with alkaline water. Such a result is consistent with the sedimentation behaviour Fig. 3(a).

\section{MICROSTRUCTURAL BEHAVIOUR OF KAOLIN CLAY WITH ACTIVE AND DEACTIVATED \\ EDGE-TO-FACE ATTRACTIVE INTERACTION}

If the electrochemical interactions induced by the different pore waters have an effect on the macroscopic behaviour of clays, such an effect must be associated with a different microstructure (i.e. different particle arrangements and/or different inter-particle forces). Observation of PSD should therefore help in understanding the different microstructures and their interplay with macroscopic behaviour.

The PSD of samples prepared with acidic water and alkaline water was measured to investigate the role of edge-to-face attraction. These samples were consolidated to two different vertical stresses $(70 \mathrm{kPa}$ and $2220 \mathrm{kPa}$, respectively) and then unloaded (Figs 4(a) and 4(b)). Samples were then removed from the oedometer and tested by MIP and SEM.

For the samples circled in Fig. 4 the PSD is reported in Figs 5(a) and 5(b), respectively. The samples reported in Fig. 5 were termed 'url' because unloading was carried out under drained conditions and the samples therefore lay on the unloading-reloading line (url) at the end of the oedometer test.

Two different classes of pores may be identified in the PSD. There is a 'dominant' part of the PSD, which is centred around the modal value approximately symmetrically in a log-scale, whereas the 'tail' part of the distribution is associated with the biggest pores. Essentially no difference can be detected in the dominant part of the PSD for samples prepared with acidic and alkaline water. In contrast, the tail part of the PSD is significantly affected by the $\mathrm{pH}$ of the pore water. In particular, the tail part of the PSD almost disappears in the sample prepared with alkaline water $(\mathrm{pH}=9)$.

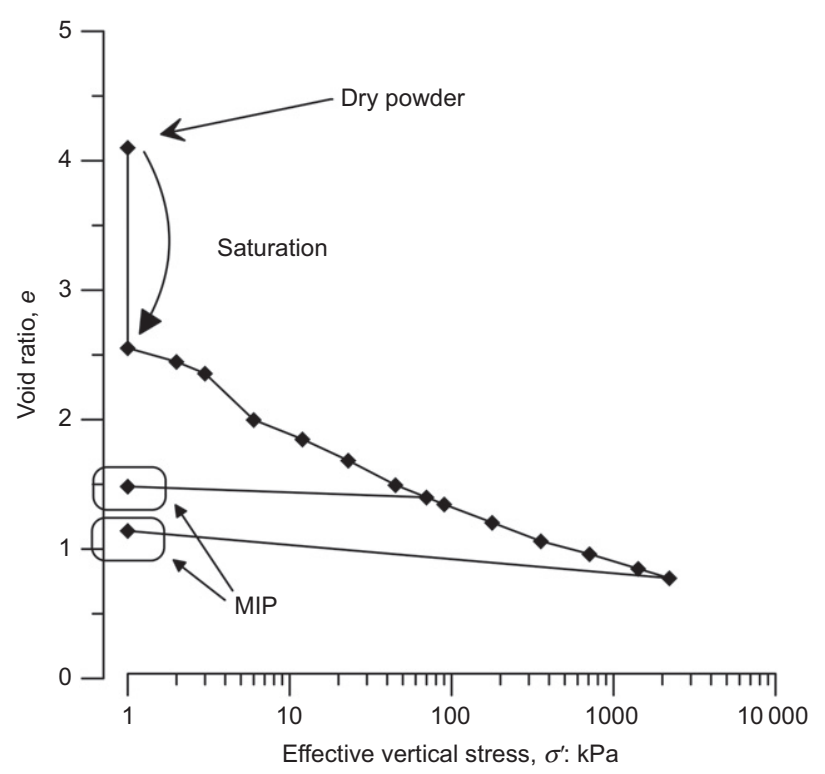

(a)

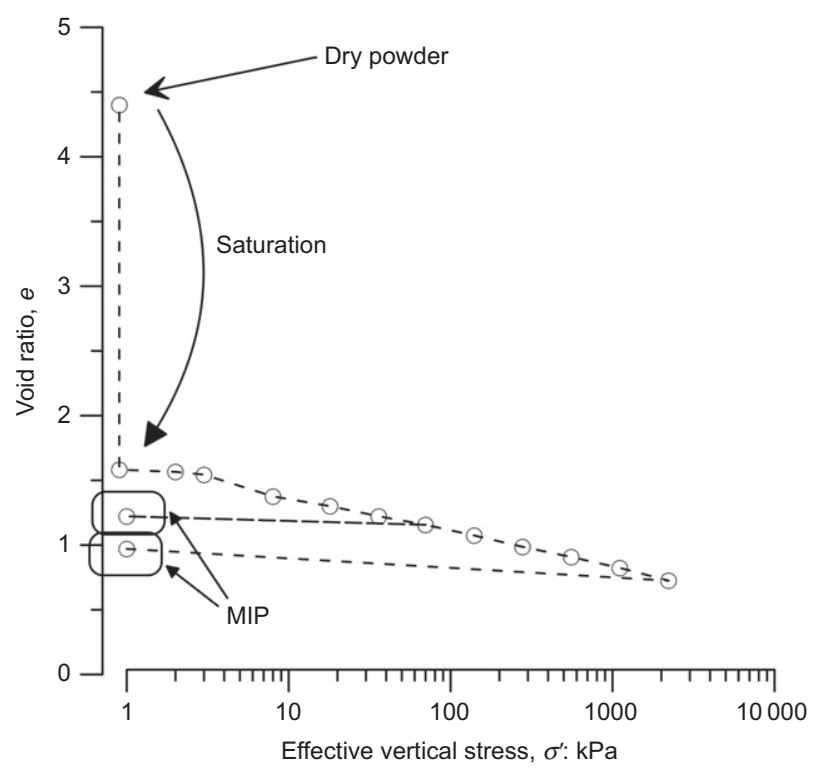

(b)

Fig. 4. One-dimensional sample preparation paths for MIP: (a) acidic water; (b) alkaline water

The alkaline water transforms the charge of the particle edge from positive to negative; in other words, the edge-to-face contact that exists when the pore water is acidic $(\mathrm{pH}=4)$ and the particle edge is positively charged is 'deactivated' by the alkaline water. The fact that the tail part of the PSD disappears in alkaline pore water therefore may indicate that such a class of pores is the one controlled by the edge-to-face attractive interaction.

Two main conclusions therefore can be drawn from Fig. 5 concerning the two classes of pores detected.

(a) Dominant part of PSD: these pores are centred around the modal value and are not controlled by the presence of the edge-to-face attraction.

(b) Tail part of the PSD: these pores have a pore size greater than the pore size of the modal value and are controlled by the presence of the edge-to-face attraction.

Figure 6 compares SEM images of two samples, prepared with alkaline (Fig. 6(a)) and acidic (Fig. 6(b)) pore water. In 


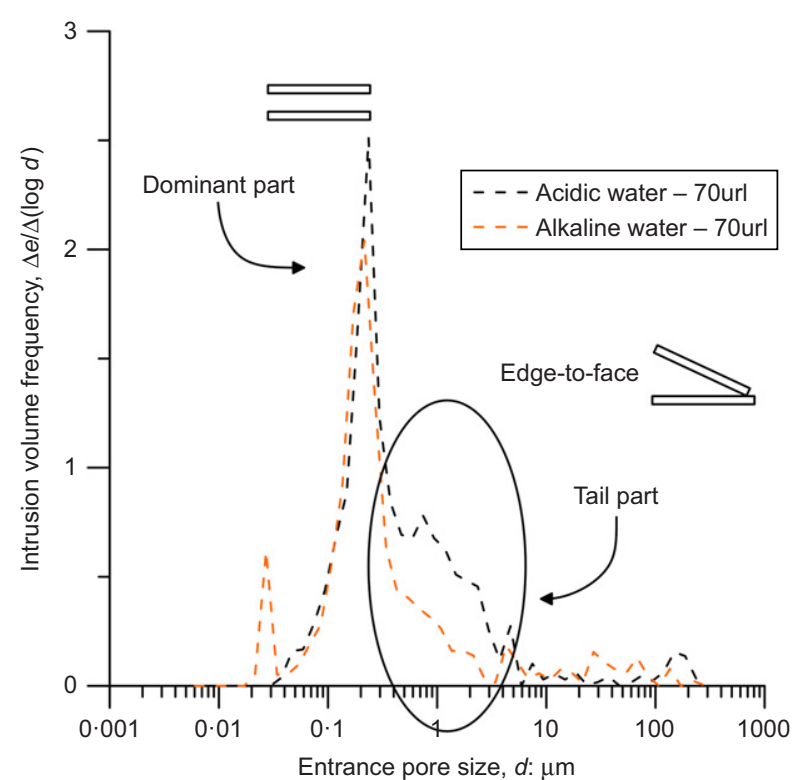

(a)

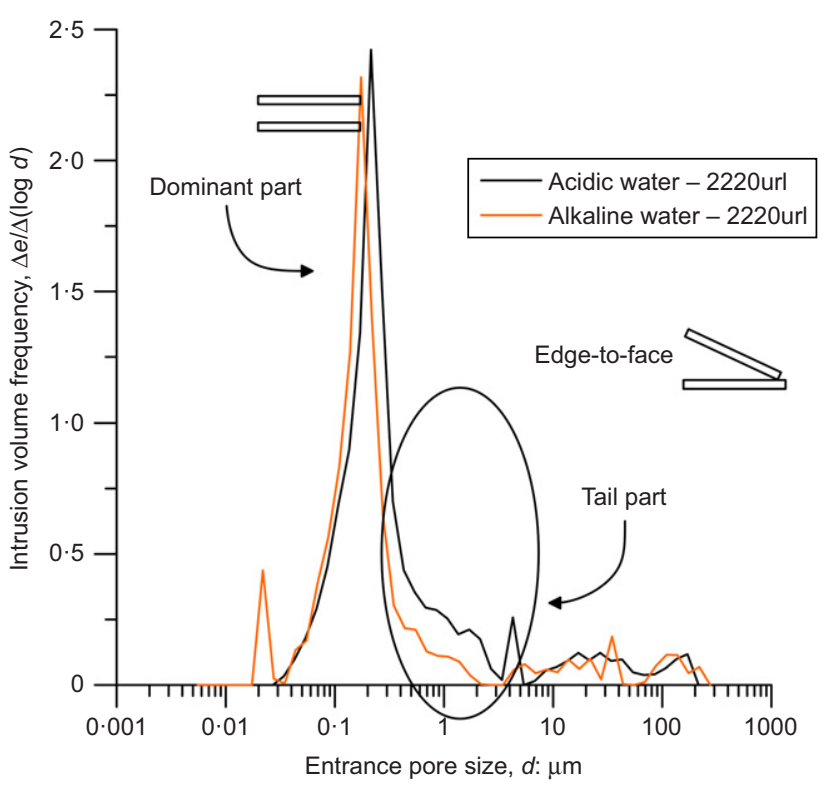

(b)

Fig. 5. Pore size distribution and cumulative intrusion void ratio of consolidated kaolin saturated with acidic water and alkaline water: (a) $70 \mathrm{kPa}$; (b) $2220 \mathrm{kPa}$

the figures, the alkaline samples show an ordered and anisotropic particle configuration. All the particles appear to be parallel to each other and no large pores can be noticed. On the other hand, the sample prepared with acidic water exhibits a much more disordered particle configuration and larger pores can be detected. It can be observed that particles are either in non-contact configuration or in contact configuration (associated with edge-to-face contact). The contact configuration is the one that appears to generate the larger pores.

\section{MICROSTRUCTURAL BEHAVIOUR OF KAOLIN CLAY AT DIFFERENT INTER-PARTICLE COULOMBIAN FORCES}

To investigate the effect of the intensity of the interaction forces, 1D compression tests were carried out on samples prepared with fluids having different dielectric permittivity.
Figure 7 shows the compression curves of specimens saturated with water, acetone and air. When the pore fluid is changed from air to acetone or from air to water, the inter-particle repulsive forces are expected to reduce (Fig. 2). Indeed, at the same initial stress $(1 \mathrm{kPa})$, the sample with water as pore fluid attained a void ratio $(e=2 \cdot 6)$ much lower than the sample with acetone as pore fluid $(e=3 \cdot 5)$, which in turn had a lower void ratio than the sample with air as pore fluid $(e=4 \cdot 1)$. This difference is maintained along the first loading as kaolin mixed with water always shows a smaller void ratio than acetone and air.

Upon unloading, rebound occurred for the three samples. The 'reversible' volume change is larger for kaolin saturated with water, intermediate for kaolin saturated with acetone and smaller for kaolin saturated with air. Such a trend is opposite to the one given by the Coulomb's force in equation (1), as if electrochemical forces did not control the rebound or if not all the pores are involved to the same extent during rebounding.

\section{Pore size distribution}

The PSD of the samples having different pore fluids was investigated by way of the MIP. Figs 8(a) and 8(b) show the PSDs of samples saturated with air and acetone, respectively. It can be noted that the PSD pattern is similar. Air-saturated and acetone-saturated samples show a mono-modal size distribution, the dominant part of the PSD remains the same regardless of the applied stress, and the tail part of the PSD reduces in size and frequency when the vertical stress is increased. These features were also observed in watersaturated samples (Figs 5(a) and 5(b)) suggesting that the same micro-mechanisms occur for the three pore fluids.

A comparison of the PSD of samples saturated with the three different pore fluids at the same vertical stress is reported in Fig. 9(a) (70 kPa vertical stress) and Fig. 9(b) (2220 kPa vertical stress). There are two major points that are worth observing.

(a) The modal pore size of the three samples is shifted as the pore fluid is changed. Modal pore size of water-saturated samples is smaller than the acetone-saturated samples, and then the air-saturated samples. This can be taken as evidence that the particle distance in the non-contact configuration is controlled by the electrical repulsion existing between the particle faces, which, in turn, depends on the dielectric permittivity of the fluid. It is worth noticing that not only the modal pore size but the whole PSD is shifted.

(b) The tail part of the PSD is shifted both in terms of pore size and frequency, with the higher pore size and frequency recorded for the air-saturated samples. For air-saturated samples the pore volume associated with edge-to-face contact is bigger than that associated with acetone-saturated samples, and in turn bigger than the pore volume associated with water-saturated samples.

\section{Scanning electron microscopy images}

Images of a sample consolidated in water to $70 \mathrm{kPa}$, as already reported in Fig. 6, are compared with images of a sample consolidated to $70 \mathrm{kPa}$ in air in Fig. 10. Particles in a face-to-face configuration and particles in edge-to-face contacts can be observed in both air-saturated and watersaturated samples. However, pore volumes generated by the edge-to-face contact in the air-saturated (dry) sample appear bigger, which is consistent with the MIP data in Fig. 9. 

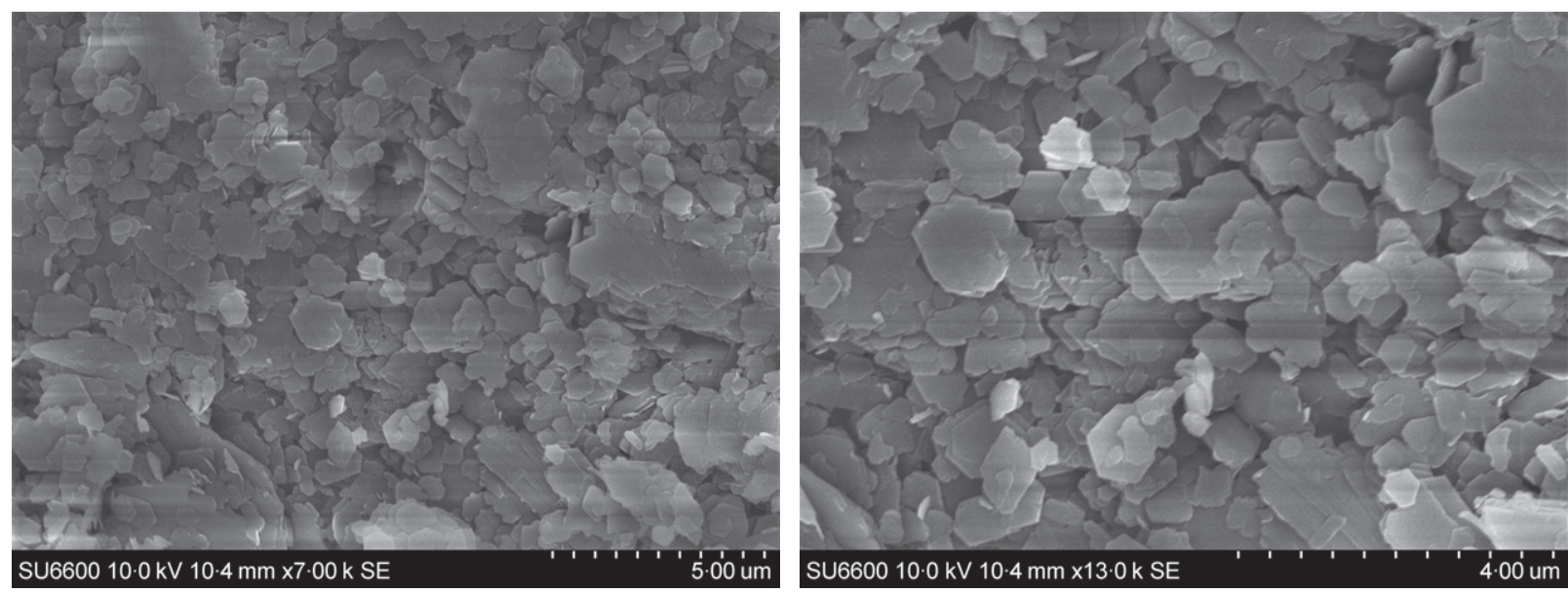

(a)
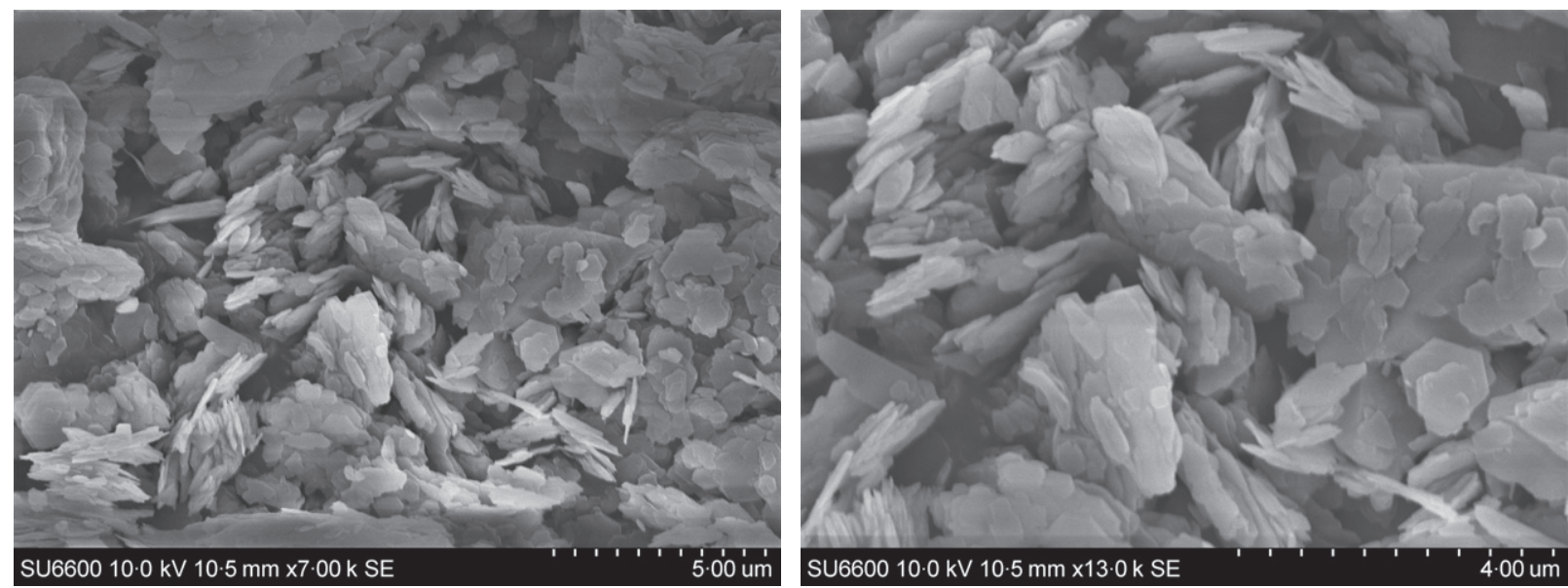

(b)

Fig. 6. SEM images on different samples consolidated after at $70 \mathrm{kPa}$ : (a) prepared with alkaline water; (b) prepared with acidic water

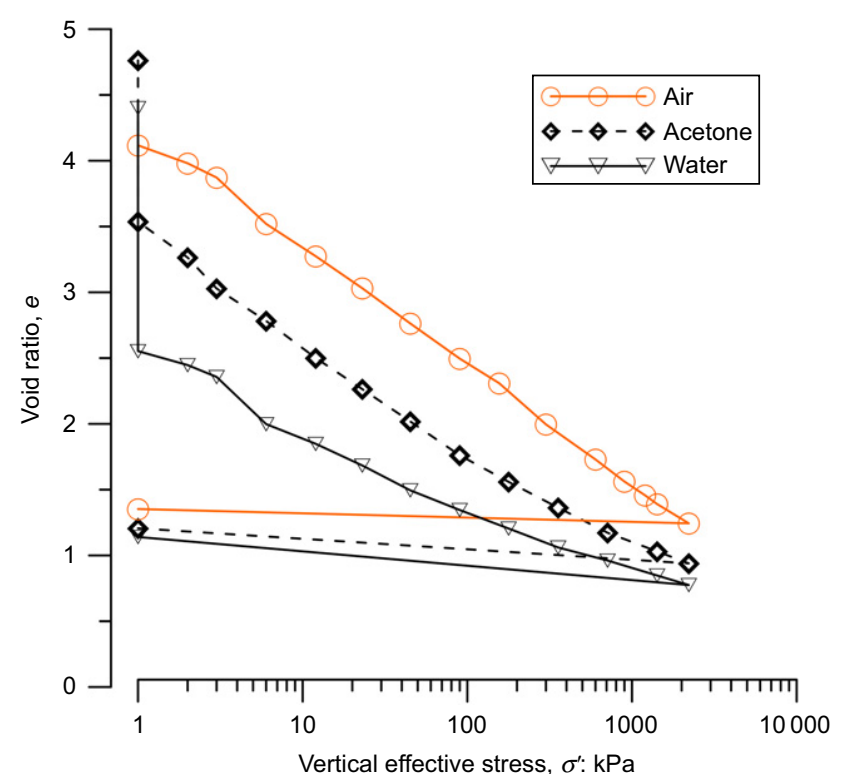

Fig. 7. One-dimensional consolidation of kaolin clay sample saturated with three different fluids

\section{MICROSTRUCTURAL BEHAVIOUR OF KAOLIN CLAY ALONG REVERSIBLE AND NON-REVERSIBLE COMPRESSION PATHS}

Figure 11(a) shows the compression curves of samples reconstituted from slurry (acidic mixture), compressed to 70 or $2220 \mathrm{kPa}$ and then unloaded. For each vertical stress (70 and $2220 \mathrm{kPa})$, two different samples were prepared, one lying on the normal consolidation line (ncl) and the other one on the unloading-reloading line (url). To test samples on the ncl, samples need to be removed from the oedometer by preventing any rebound. To this end, once the desired vertical stress was attained, the sample was unloaded quickly to generate undrained conditions.

To achieve undrained conditions, the time taken to remove the load should be significantly shorter than the time of consolidation. Because the time required to remove the load cannot be reduced beyond a certain limit, the time of consolidation was increased by increasing the drainage length. A sheet of para-film was placed at the bottom of the sample in order to seal the bottom porous stone. As water flow was permitted only at the top of the sample, the drainage length could be doubled and the consolidation time could then be incremented by four times. The undrained unloading was then performed in one single step, the vertical stress was reduced to $1 \mathrm{kPa}$ and the sample was rapidly removed from the oedometer. Once removed from the oedometer the sample was stored in a sealed bag to prevent any water loss until freeze-drying was performed.

For the url samples, a drained unloading was performed following the same procedure described in the previous section. Fig. 11(a) shows the location of the samples tested in the MIP on the normal compression and unloading curves.

Figure 12 shows the pore size evolution upon unloading of samples consolidated to $70 \mathrm{kPa}$ and $2220 \mathrm{kPa}$. It can be observed that the unloading does not change the shape of the 


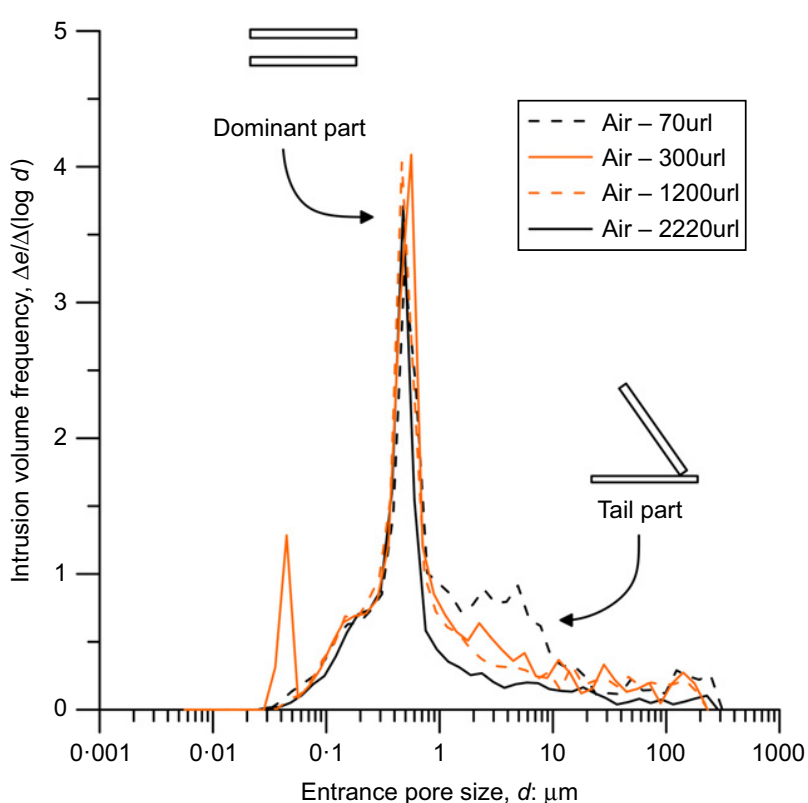

(a)

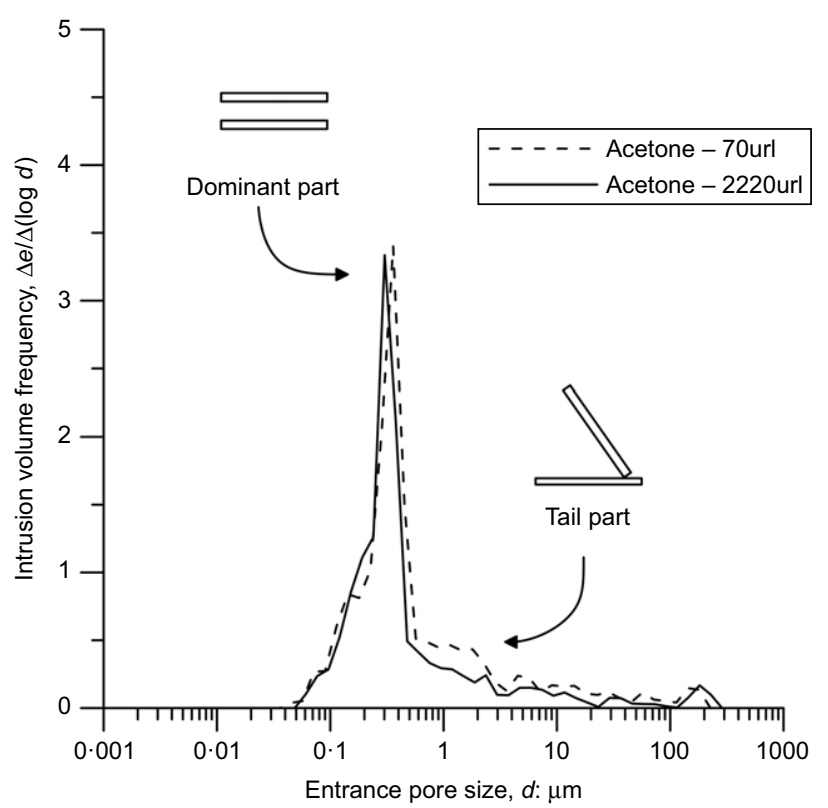

(b)

Fig. 8. Pore size distribution at different vertical stress: (a) air; (b) acetone

PSD. However, a clear shift rightward is observed upon unloading; in other words, the whole distribution shifts towards larger pore diameters. This is observed for both samples, loaded to $70 \mathrm{kPa}$ and $2220 \mathrm{kPa}$, respectively, and then unloaded.

An additional series of samples, consolidated to $70 \mathrm{kPa}$, $178 \mathrm{kPa}, 711 \mathrm{kPa}$ and $2220 \mathrm{kPa}$, was prepared. All samples were loaded and unloaded under drained conditions (url). The state of these samples is shown in Fig. 11(b). In Fig. 11(b) an additional sample called 'slurry' is reported. This sample was tested in the MIP just after imbibition of the clay in the oedometer cell. Fig. 13 shows the pore size evolution associated with the accumulation of plastic deformation (samples have all been brought to $1 \mathrm{kPa}$ after being pre-consolidated to different vertical stresses). The dominant part of the PSD remains the same over the entire stress range, which goes from slurry (no load applied) to the maximum vertical stress of $2220 \mathrm{kPa}$. In contrast, the

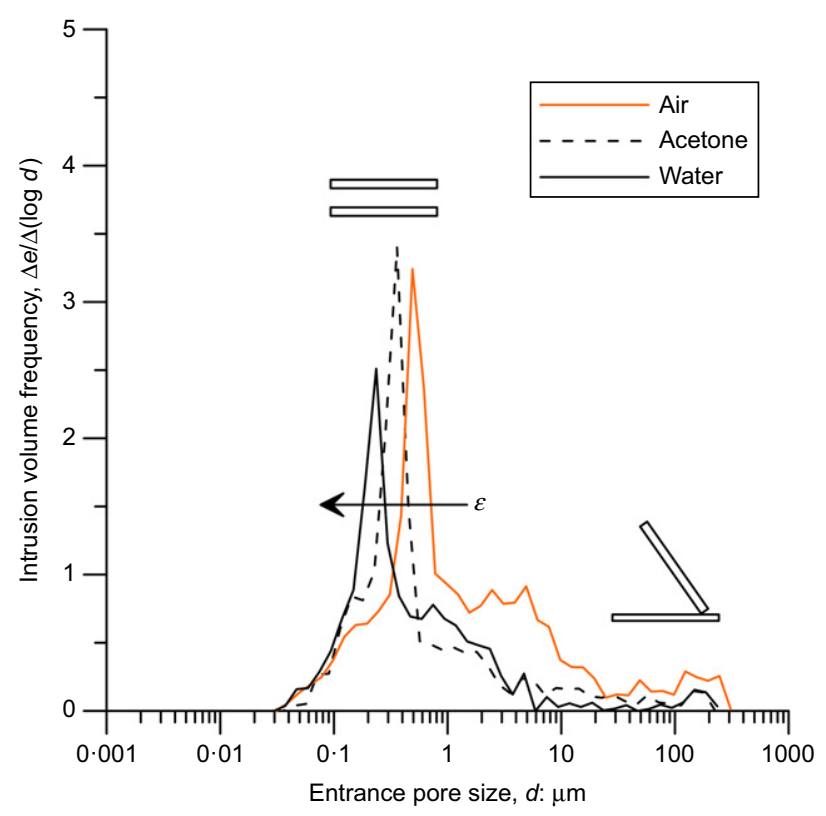

(a)

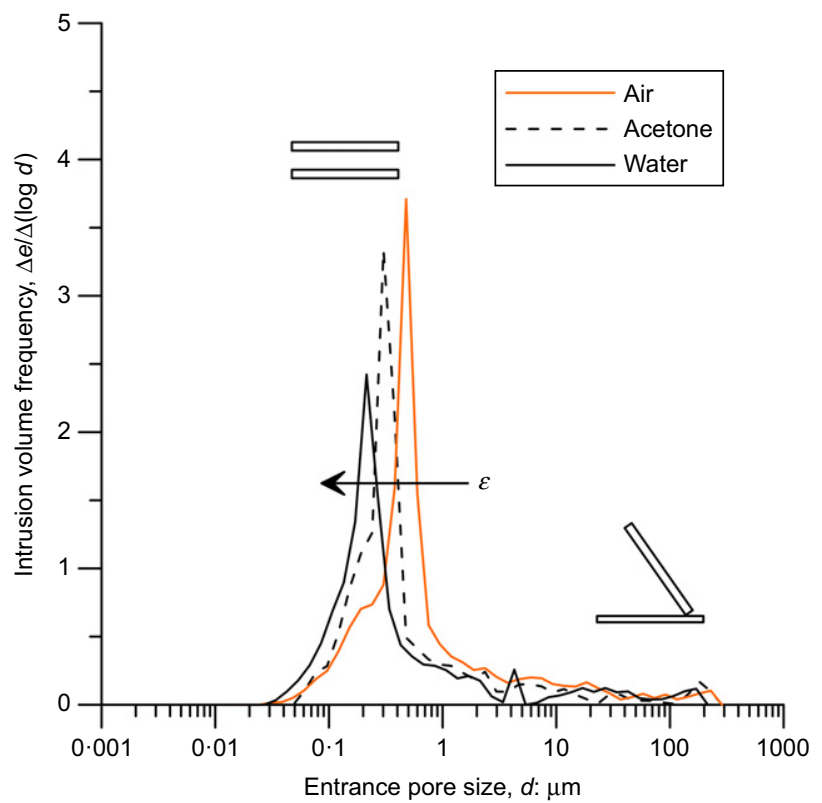

(b)

Fig. 9. Pore size distribution of samples consolidated at (a) $70 \mathrm{kPa}$ and (b) $2220 \mathrm{kPa}$ having different pore fluids

volume change associated with yielding is clearly visible in the evolution of the tail porosity. The higher the consolidation stress, the smaller is the frequency at any given pore diameter in the tail.

The evolution of the tail upon loading and unloading can be better appreciated when the cumulative pore volume is considered. Fig. 14(a) shows the cumulative pore volume of the samples in Fig. 13. To compare the dominant parts of the PSD of the different samples, the modal value and the corresponding cumulative void ratio, $e^{*}$, were selected as characteristic values of the dominant part.

The total volume associated with the dominant part of the PSD remains essentially unaltered regardless of the vertical stress. On the other hand, starting from slurry, any increase in the vertical stress causes a significant reduction of the total volume associated with the tail part of the PSD. As all samples lie on a url line at $1 \mathrm{kPa}$, any difference $\Delta e$ in 

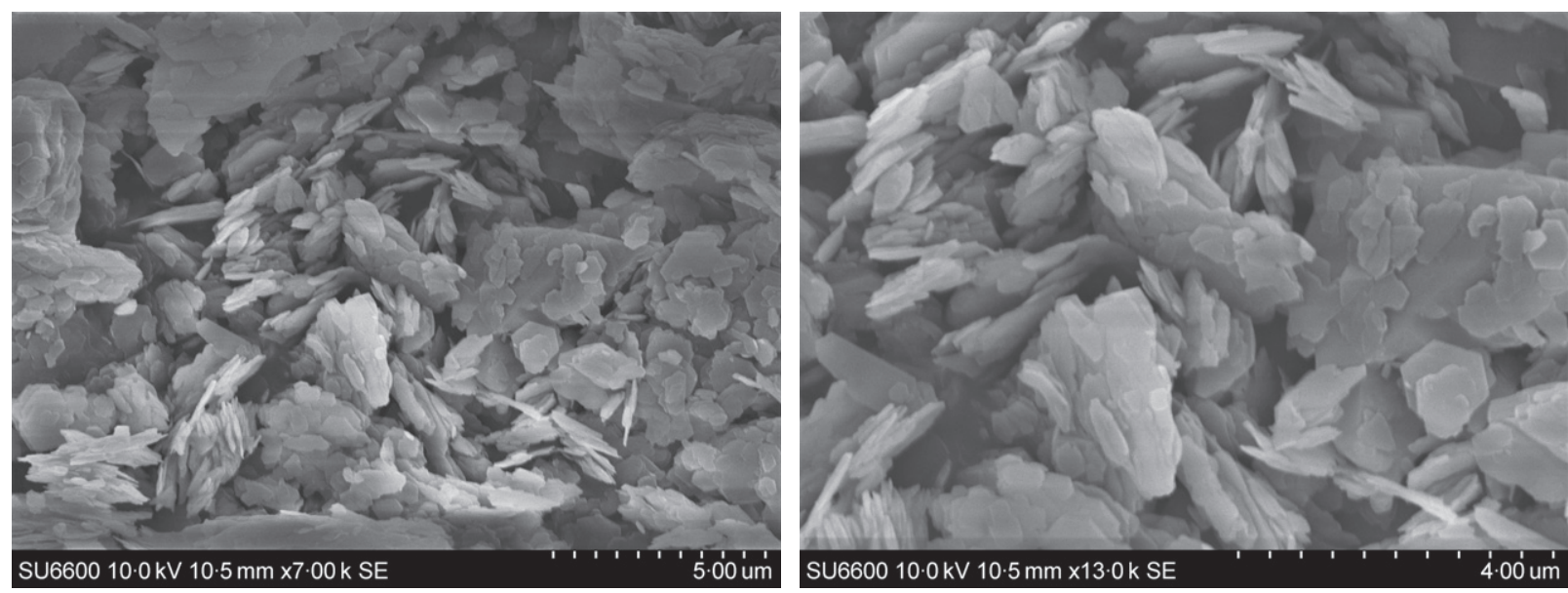

(a)
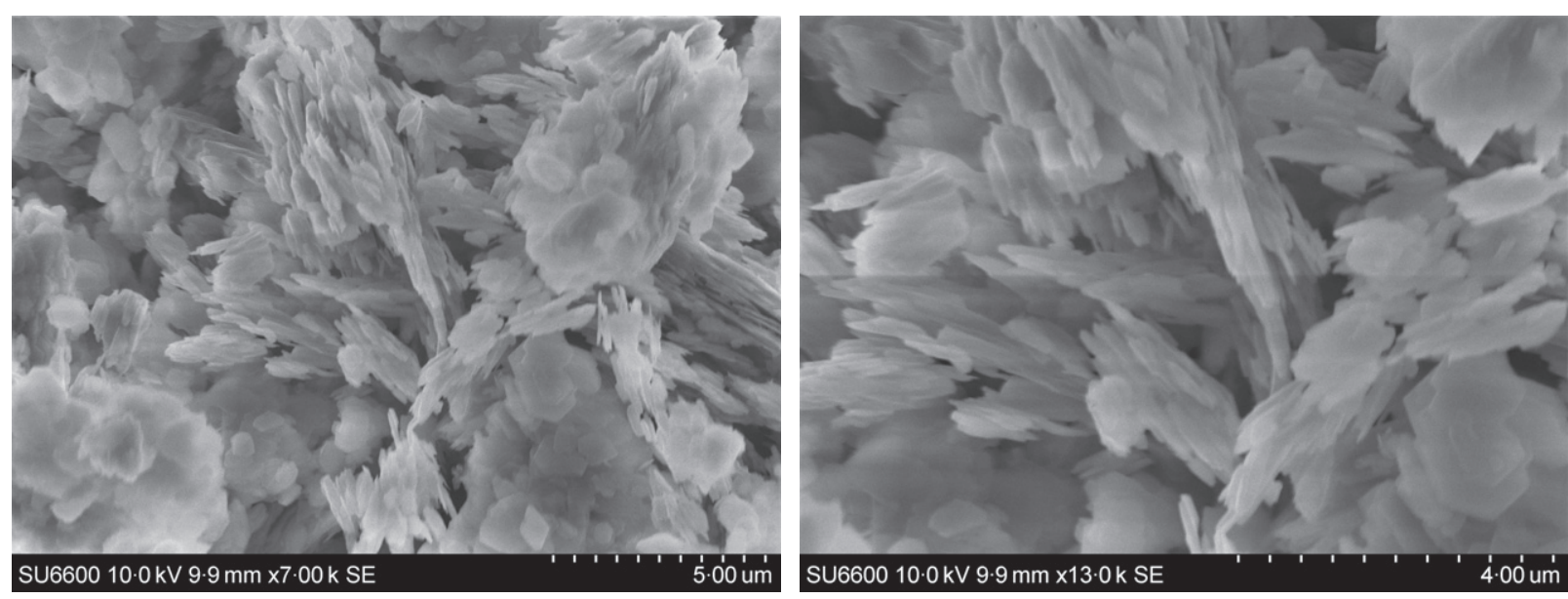

(b)

Fig. 10. (a) Sample consolidated in water $(70 \mathrm{kPa})$. (b) Sample consolidated in air (dry powder) $(70 \mathrm{kPa})$

void ratio is associated with plastic deformation accumulated when moving from one pre-consolidation stress to another.

In order to visualise the classes of pores exhibiting reversible and non-reversible behaviour, it is convenient to compare the cumulative pore volume (Fig. 14(b)) and the PSD (Fig. 14(c)) of three samples. Figs 14(b) and 14(c) show the comparison between sample (labelled 1) consolidated to $70 \mathrm{kPa}$ and unloaded under drained conditions (water 70url), sample (labelled 2) consolidated to $2220 \mathrm{kPa}$ and unloaded under undrained conditions (water $-2220 \mathrm{ncl}$ ) and sample (labelled 3) consolidated at $2220 \mathrm{kPa}$ and unloaded under drained conditions (water - 2220url). The state of these three samples is visualised at the bottom right-hand corner of Fig. 14(b).

The change in volume between samples 1 and 2 is a sum of a reversible and non-reversible deformation (stress path is ncl + url). The change in volume between samples 2 and 3 is only associated with reversible deformation (stress path on url). Therefore, the change in volume between sample 1 and 3 is the non-reversible deformations accumulated between the two different pre-consolidation stresses.

Although reversible volume change (associated with the path 2-3) affects both the dominant part and the tail part of the PSD, it can be observed that the total reversible volume change is essentially dominated quantitatively by the volume change of the pores belonging to the tail part of the PSD. As a first approximation, the reversible volume change can therefore be associated with the volume change of such 'tail' pores.
Two main conclusions can therefore be drawn from Figs 13 and 14

(a) Reversible behaviour affects both the pores belonging to the dominant part and to the tail part of the PSD; that is, all pores are involved in the 'elastic' deformation of the clay. Nonetheless, volume change of pores associated with the contact configuration is the most relevant quantitatively.

(b) Pores of the dominant part of the PSD of samples in the unloaded state remain unaltered regardless of the different pre-consolidation stress previously attained. Only the volume associated with the tail part of the PSD reduces when plastic changes occur. Non-reversible volume change therefore appears to involve only the tail part of the PSD.

\section{MICROMECHANICAL CONCEPTUAL MODEL FOR NON-ACTIVE CLAY}

Two classes of pores have been experimentally identified and associated with two distinct particle configurations

(a) non-contact configuration associated with pores belonging to the dominant part of the PSD

(b) contact configuration associated with pores belonging to the tail part of the PSD.

For each particle configuration, micro-mechanisms assumed to occur along loading (irreversible) and unloading 


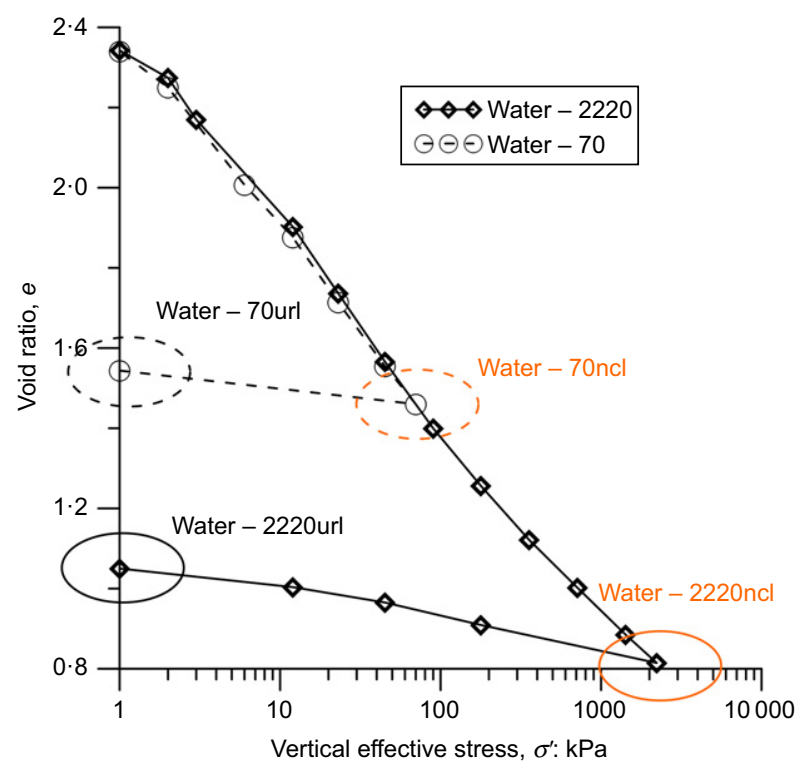

(a)

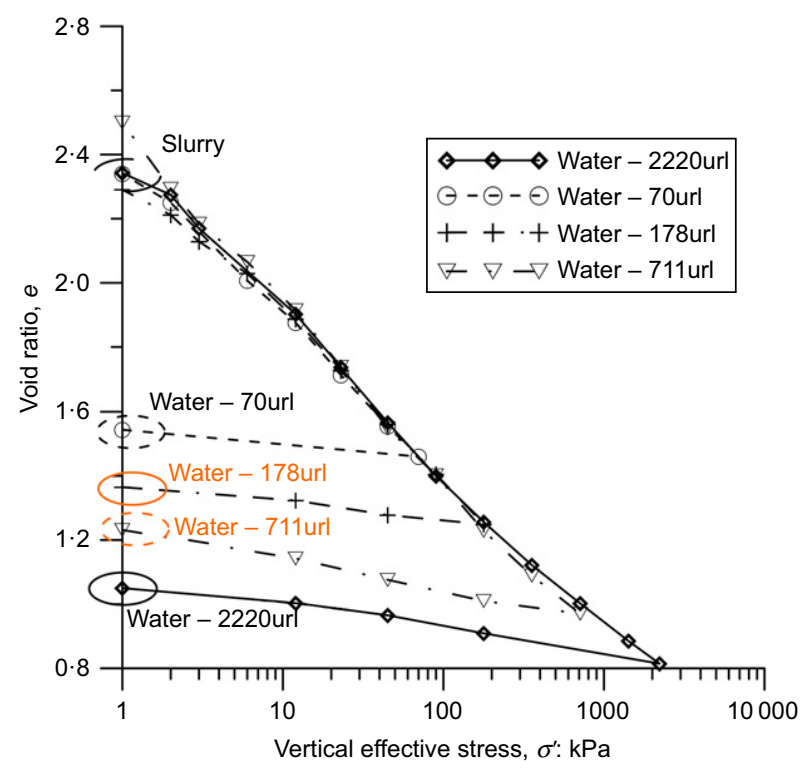

(b)

Fig. 11. Mono-dimensional compression on kaolin mixed with water. Circles indicate samples tested in MIP. (a) Samples compressed, respectively, to 70 and $2220 \mathrm{kPa}$ and then unloaded. (b) Samples compressed, respectively, to 70, $178 \mathrm{kPa}, 711 \mathrm{kPa}$ and $2220 \mathrm{kPa}$ and then unloaded

(reversible) paths can be summarised as follows (Fig. 15). Non-contact configuration is only subjected to reversible deformation (Figs 12 and 13). The inter-particle applied force is counterbalanced by repulsion forces upon reversible compression paths. Particles move closer or apart with increases and decreases in applied external stress, respectively.

On the other hand, contact configuration gives rise to both reversible (Fig. 12) and non-reversible mechanisms (Fig. 13).

The following micro-mechanism can be tentatively figured out for the contact configuration. Upon reversible paths, particles in contact configuration tilt with respect to each other around the contact (from A to B in Fig. 15). As in the non-contact configuration, the relative tilt is controlled by the repulsive forces existing between particles. The contact point could be figured out as a frictional constraint in the horizontal direction (parallel to the particle face) and a unilateral constraint in the vertical direction (orthogonal to

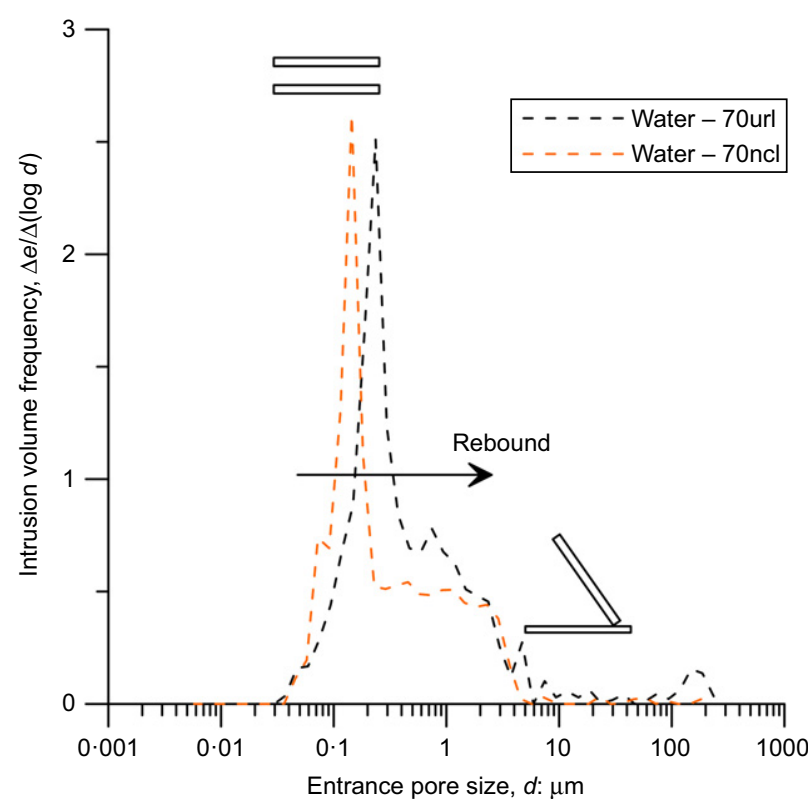

(a)

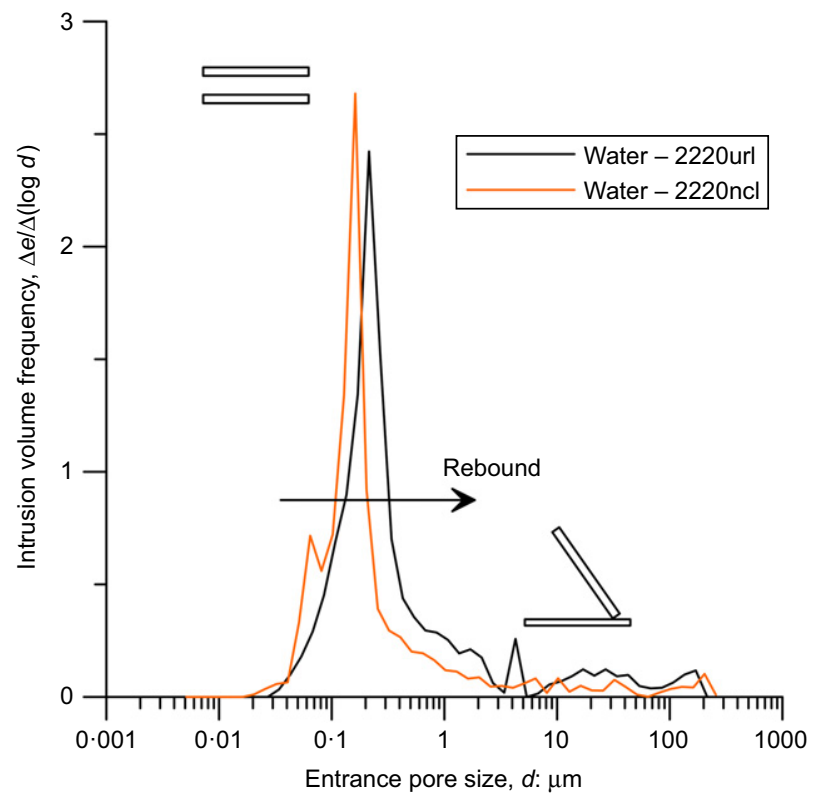

(b)

Fig. 12. Pore size evolution during rebound: (a) $70 \mathrm{kPa}$; (b) $2220 \mathrm{kPa}$

the particle face) (Fig. 16(b)). The former is controlled by a particle-to-particle friction mechanism, whereas the latter prevents penetration between two particles and leaves the two particles free of moving apart. If the threshold frictional shear force is not exceeded, the constraint behaves as a simple bearing in the horizontal direction (no relative movement of the contact).

Upon irreversible paths, the loss of contact could be associated with either a tangential sliding or normal detachment. If the mobilised contact shear force exceeds the threshold frictional resistance or the mobilised normal force changes from compressive to tensile, the inter-particle contact is disengaged and plastic deformation occurs (from B to $\mathrm{C}$ in Fig. 15). An example of a possible mechanism of disengagement for a given particle configuration and applied external force is discussed in the Appendix. In this case, for example, tangential slippage is the more likely to occur with respect to normal disengagement. 


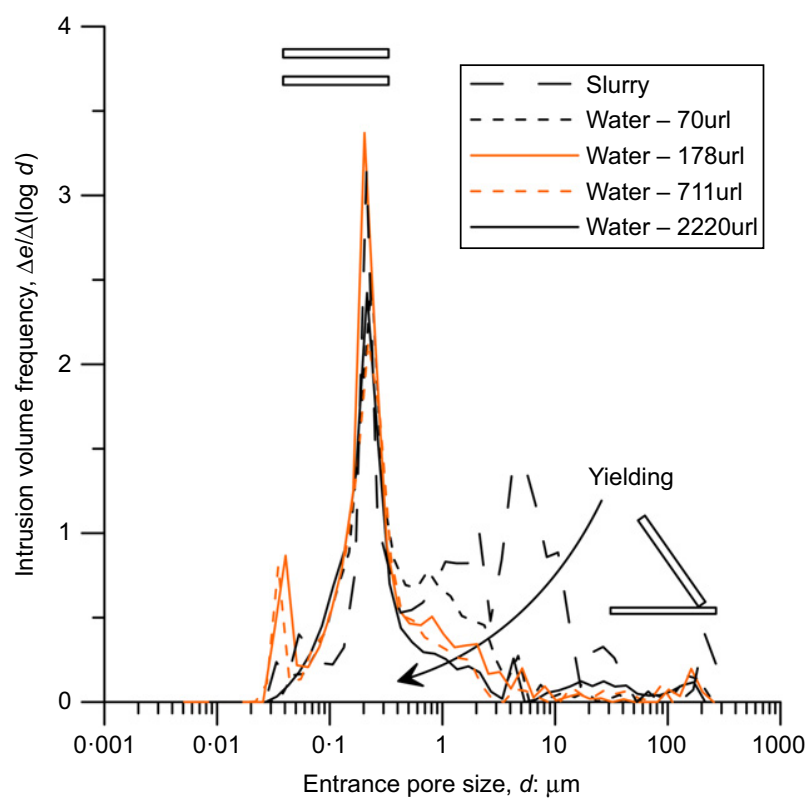

Fig. 13. Pore size evolution during consolidation at different maximum vertical stress
Once a contact has been disengaged, it is speculated that particles tend to dispose in a sub-parallel configuration and, from this point on, the mechanical response of these particles becomes exclusively reversible (non-contact configuration).

In summary, plastic deformations are here assumed to be mainly associated with the disengagement of the interparticle contact, which is considered to be a non-reversible mechanism.

\section{QUALITATIVE EVALUATION OF THE CONCEPTUAL MODEL}

The conceptual micromechanical model illustrated in Fig. 15 is predisposed to be potentially implemented into a DEM model. To show the potential of a DEM implementation, an embryonic 1D discrete-element model was developed to explore whether the main features of the response at a macroscopic level could be captured by discrete-element modelling (Fig. 17).

Only particles in contact configuration were considered in the 1D model. Once particles moved from contact to non-contact configuration, the void volume associated with contact configuration was assumed to be equal to zero. This is because the 'elastic' change in volume of voids associated with a particle in non-contact configuration appears be

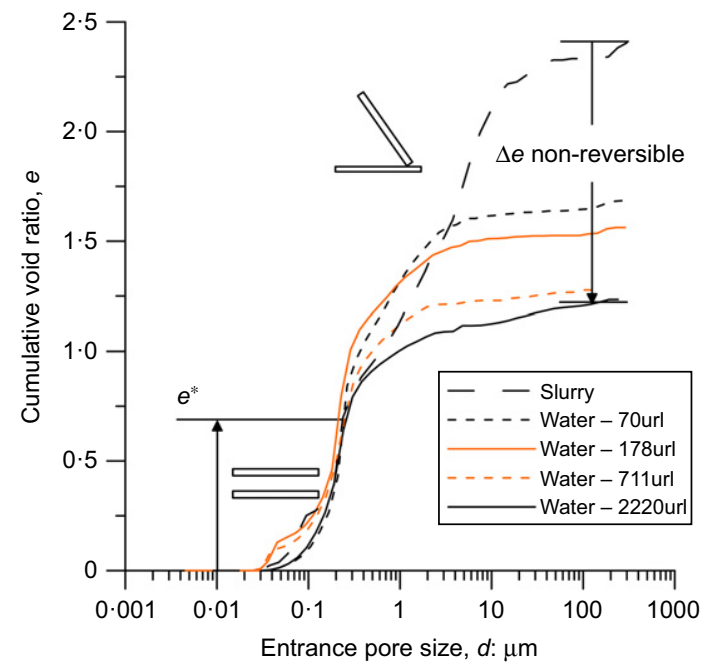

(a)

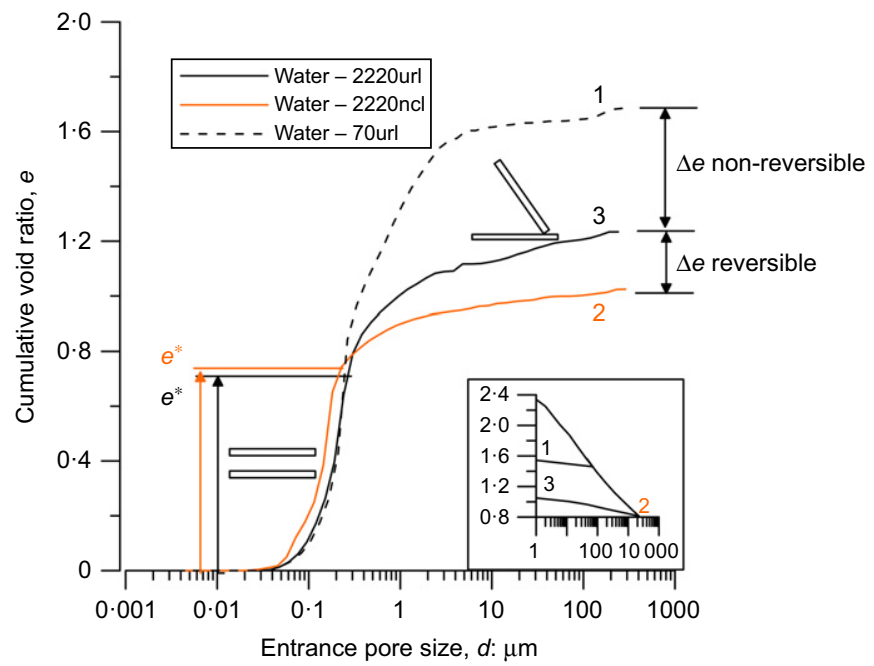

(b)

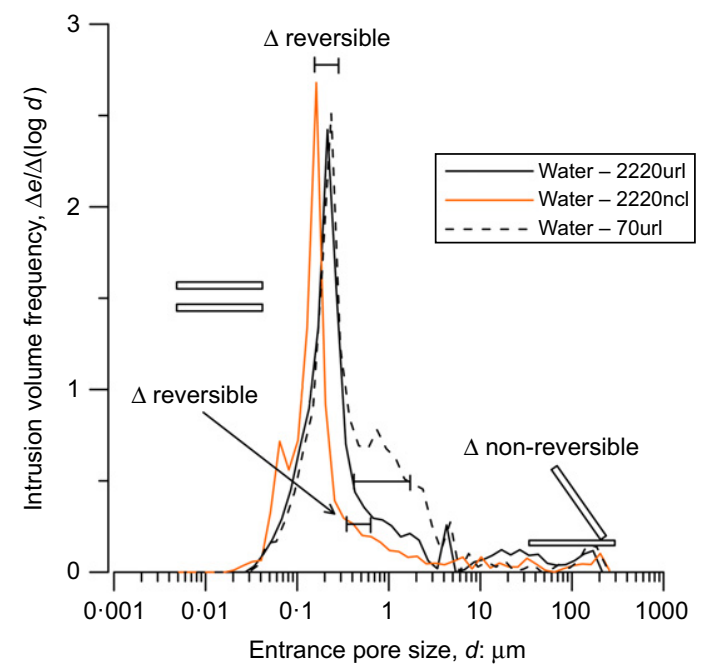

(c)

Fig. 14. Pore size for different vertical stress. (a) Cumulative void ratio for kaolin sample consolidated at different vertical stress. (b) Cumulative void ratio for kaolin samples upon a loading-unloading cycle. (c) PSD for kaolin samples upon a loading-unloading cycle 


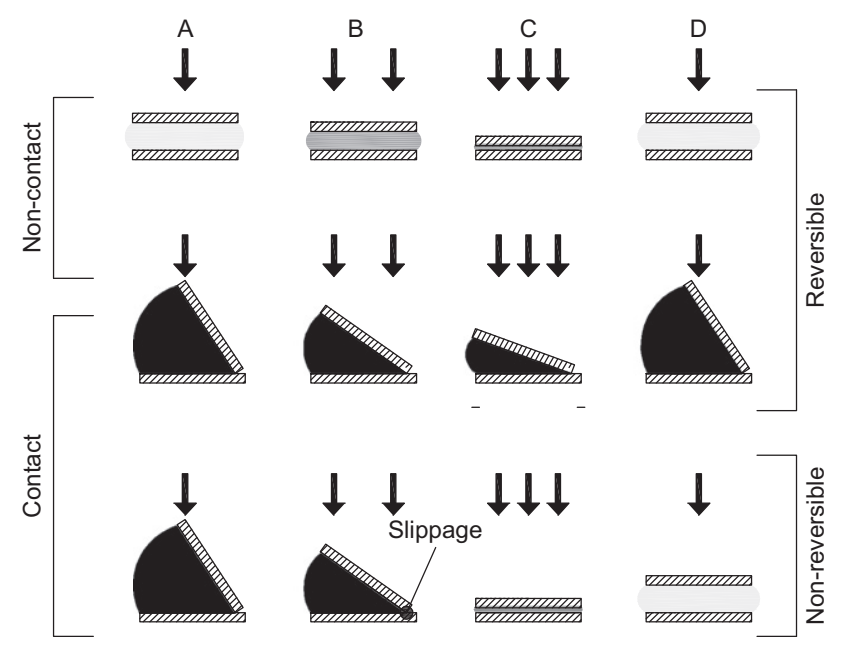

Fig. 15. Micro-mechanism associated with contact and non-contact configuration

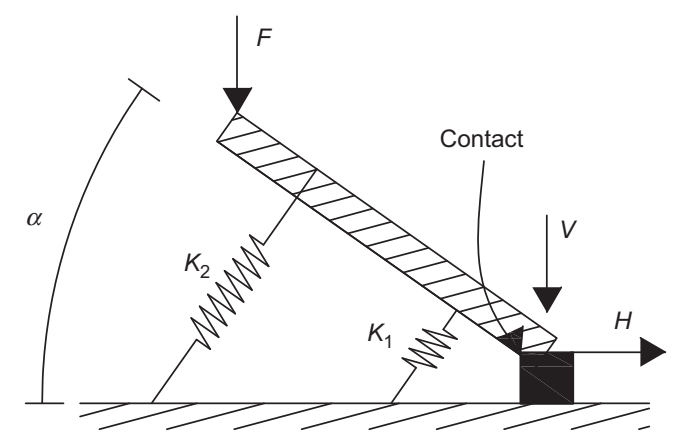

(a)

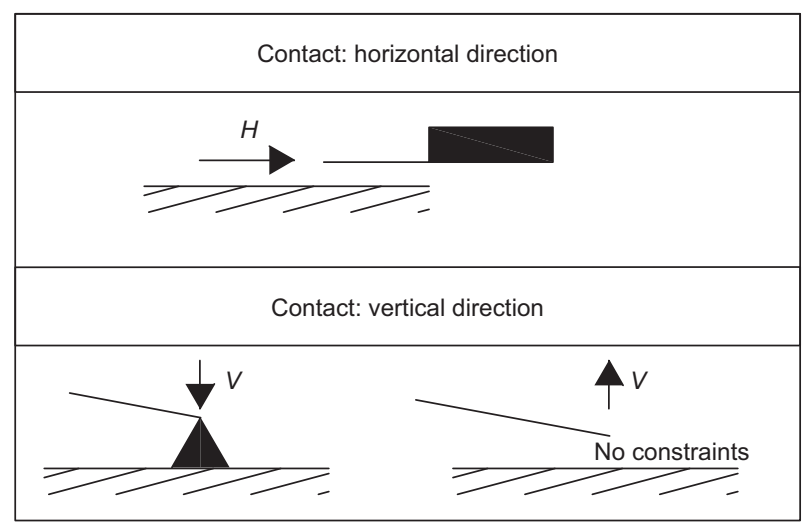

(b)

Fig. 16. Mechanical sketch of edge-to-face interaction: (a) mechanical interaction between particles; (b) constraints at particle contact

negligible compared to the 'elastic' change in volume associated with a particle in contact configuration, as shown in Fig. 14.

Particle-to-particle interaction was modelled according to Fig. 16. For a given load, the inter-particle distance is controlled by the repulsive force existing between the faces (springs $K_{1}$ and $K_{2}$ in Fig. 16). As shown by equation (1), the inter-particle repulsive force is controlled by the pore-fluid dielectric permittivity, $\varepsilon$, and the particle dimension, $a$. At the contact, perfectly plastic frictional behaviour was assumed. Once the mobilised tangential force at the contact attains the frictional resistance, slippage occurs and particles move from contact to non-contact (sub-parallel) configuration. A non-reversible volume change therefore takes place.

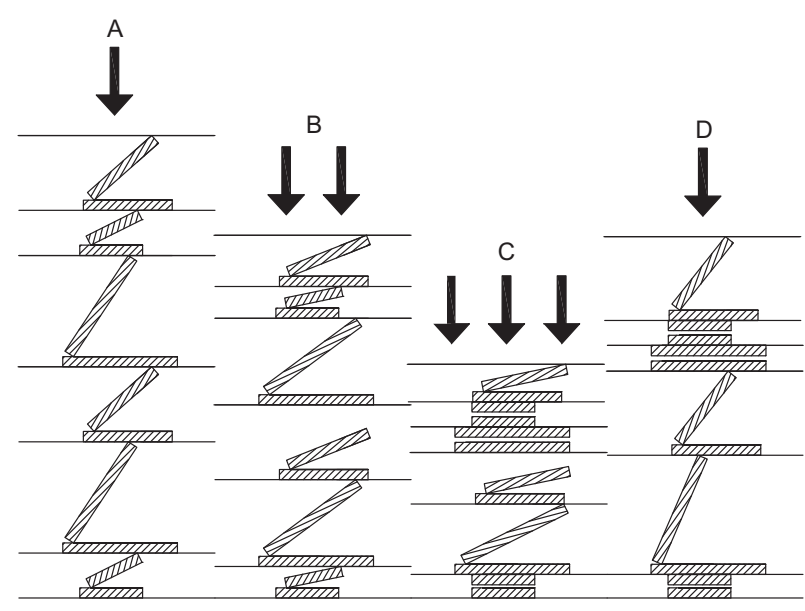

Fig. 17. Particle stacking for simulation

The model parameters are described below under the categories of geometric and inter-particle interaction parameters.

\section{Geometric parameters}

(a) $N_{\text {TOT: }}$ total number of particle pairs

(b) $t$ : thickness of the particle (m)

(c) $\bar{a}$ : mean of the distribution of the particle dimension $a$

(d) $\sigma_{a}$ : standard deviation of the distribution of the particle dimension $a$.

\section{Inter-particle interaction parameters}

(a) $\rho$ : charge density of the surface $\left(\mathrm{C} / \mathrm{m}^{2}\right)$

(b) $\varepsilon_{\mathrm{r}}$ : relative dielectric permittivity of the fluid

(c) $V_{\mathrm{EF}}$ : adhesion at the contact $(\mathrm{N})$

(d) $\mu$ : friction coefficient at the contact

\section{Void ratio}

To define the void ratio in the 1D model, the volume of voids was associated with the aperture of particle pairs ( $V_{\mathrm{v}}=a \sin \alpha$ where $\alpha$ is the tilt angle between two particles and $a$ is the length of the particle in the pair). The volume of solids was calculated based on the thickness of the particles $\left(V_{\mathrm{s}}=N_{\mathrm{TOT}} t\right)$. Particles that are moving to contact configuration were assumed not to contribute to the volume of voids any longer, but only to the volume of solids.

\section{Non-dimensional internal force}

The inter-particle distance $z$ is controlled by the nondimensional group on the right-hand side of equation (2) (derived by rearranging equation (1))

$$
\tilde{F}=\frac{F}{\left(\pi a^{2} \rho^{2} / 8 \varepsilon_{0}\right)}=\frac{1}{\varepsilon_{\mathrm{r}}}\left(\frac{z}{\sqrt{a^{2}+z^{2}}}-1\right)
$$

which allows a non-dimensional applied internal force $\tilde{F}$ to be defined.

\section{Qualitative calibration of the model}

The geometric parameters of the model parameters were assumed a priori. The particle dimension $a$ was assumed to 


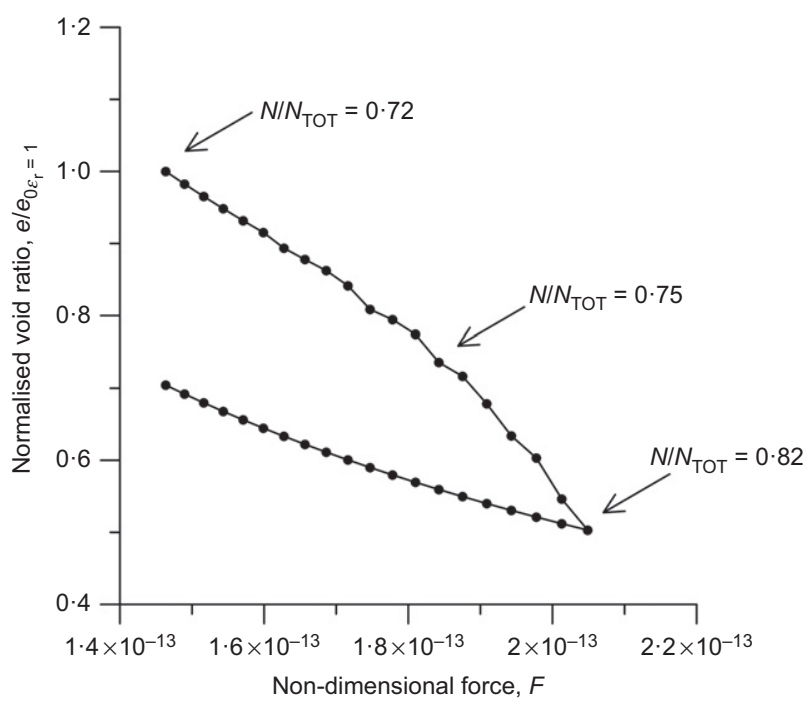

Fig. 18. Simulation of $1 \mathrm{D}$ compression for dielectric permittivity $\varepsilon_{\mathrm{r}}=1$

be distributed log-normally (average $\bar{a}=1 \mu \mathrm{m}$ and standard deviation $\sigma_{a}=0.2 \mu \mathrm{m}$ ) and the thickness of the particle was taken as $t=1 / 10 a$. The total number of particle pairs was chosen as $N_{\text {TOT }}=100$.

The friction coefficient controlling slippage at the edge-to-face contact, $\mu$, the adhesion force, $V_{\mathrm{EF}}$, and the range of loading in terms of non-dimensional force, $\tilde{F}$, were chosen to reproduce virgin loading and unloading-reloading curves that were in qualitative agreement with the experimental data as shown in Fig. 7 (for one reference relative dielectric permittivity value $\left.\varepsilon_{\mathrm{r}}=1\right)$. This simulation was obtained for

$$
\begin{aligned}
& \mu=0.36 \\
& \frac{V_{\mathrm{EF}}}{\left(\pi a^{2} \rho^{2} / 8 \varepsilon_{0}\right)}=4.6585 \times 10^{-13} \\
& 1.4635 \times 10^{-13}<F<2.0489 \times 10^{-13}
\end{aligned}
$$

The void ratio $e$ normalised to the initial void ratio $e_{0}$ is plotted against the non-dimensional force $\tilde{F}$ in Fig. 18. The yielding index is also plotted in the figure, which increases with the non-dimensional load. The yielding index is here defined by the ratio $N / N_{\text {TOT, }}$, where $N$ is the number of particle pairs yielded, when moving from a contact to a non-contact configuration.

It is worth noting that each pair that remains in contact configuration is subjected to a reversible deformation as the stiffness of the springs does not change upon loading and unloading. The overall non-reversible deformation is due to the smaller number of pairs contributing to the rebound.

\section{Simulation and discussion}

In order to explore the effect of the pore fluid dielectric permittivity on the compression behaviour of the sample, the relative dielectric permittivity $\varepsilon_{\mathrm{r}}$ was varied from 0.8 to 1.2 by leaving unchanged all the other parameters of the model (Fig. 19).

When the relative dielectric permittivity is increased, the initial void ratio (normalised with respect to the initial void ratio for the reference case $\varepsilon_{\mathrm{r}}=1$ ) decreases and the sample become less compressible, in qualitative agreement with the experimental data shown in Fig. 7.

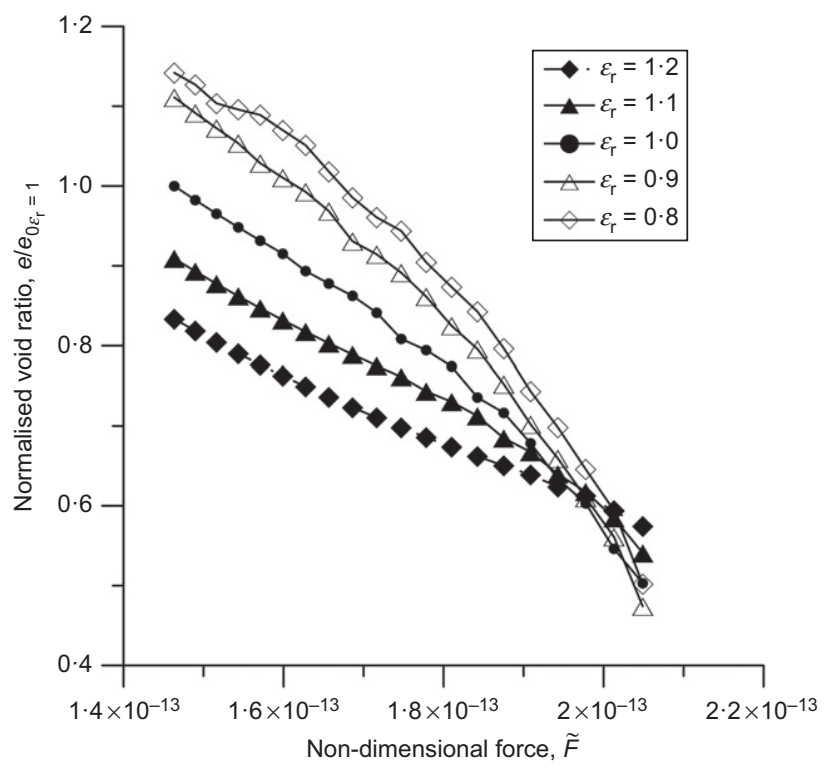

Fig. 19. One-dimensional compression test upon loading for different dielectric constant values

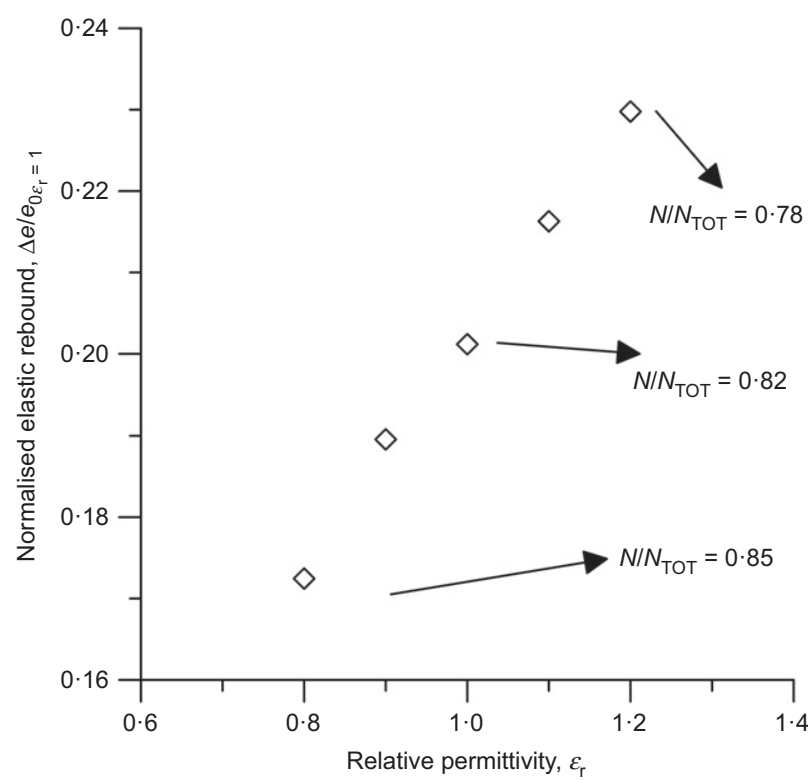

Fig. 20. Elastic rebound upon unloading for different dielectric constant values

Figure 20 shows the amount of elastic rebound. Consistently with the experimental data, the higher the dielectric permittivity, the higher is the amount of rebound. As shown in Fig. 20, the yielding index decreases as the relative dielectric permittivity increases. As a result, a higher number of particle pairs are contributing to the rebound.

\section{CONCLUSIONS}

The paper has presented an experimental investigation into the micro-mechanisms controlling the behaviour of non-active clays, in particular the mechanisms underlying reversible and non-reversible compression. Clay microstructure was investigated by way of MIP and SEM.

Samples for MIP and SEM testing were taken along both normal compression and unloading-reloading lines to characterise the evolution of clay microstructure upon non-reversible and reversible compression paths. The strategy 


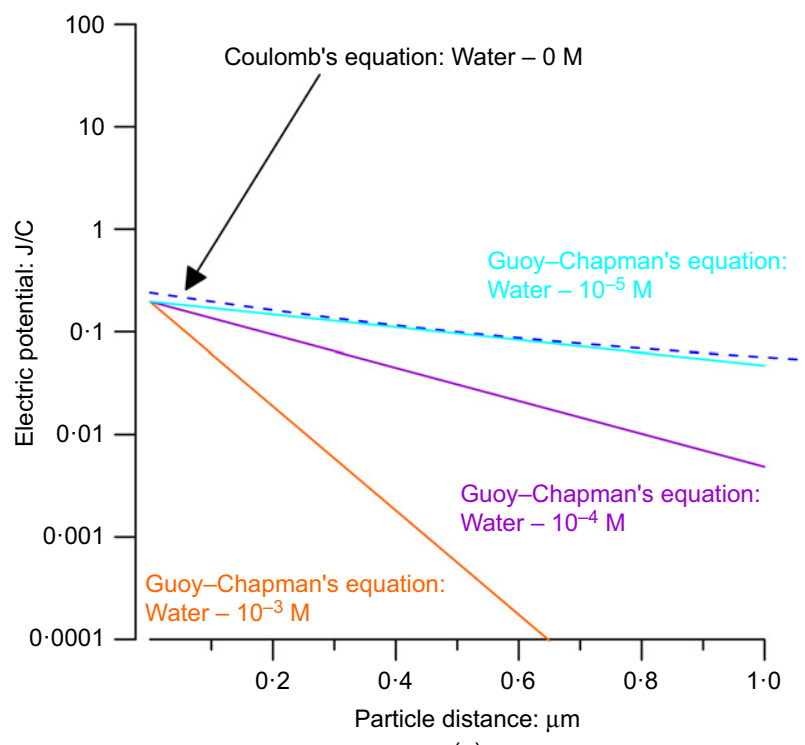

(a)

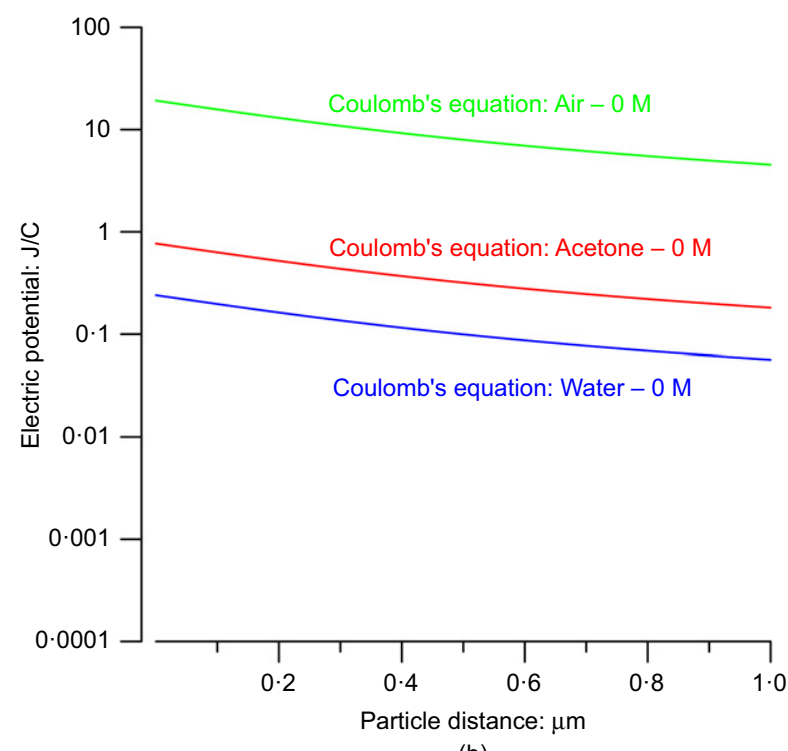

(b)

Fig. 21. Computation of electric potential for different dielectric media: (a) electric potential for sodium chloride solutions with molar concentration of $10^{-3}, 10^{-4}$ and $10^{-5} \mathrm{M}$; (b) electric potential for air, acetone and water as pore fluid

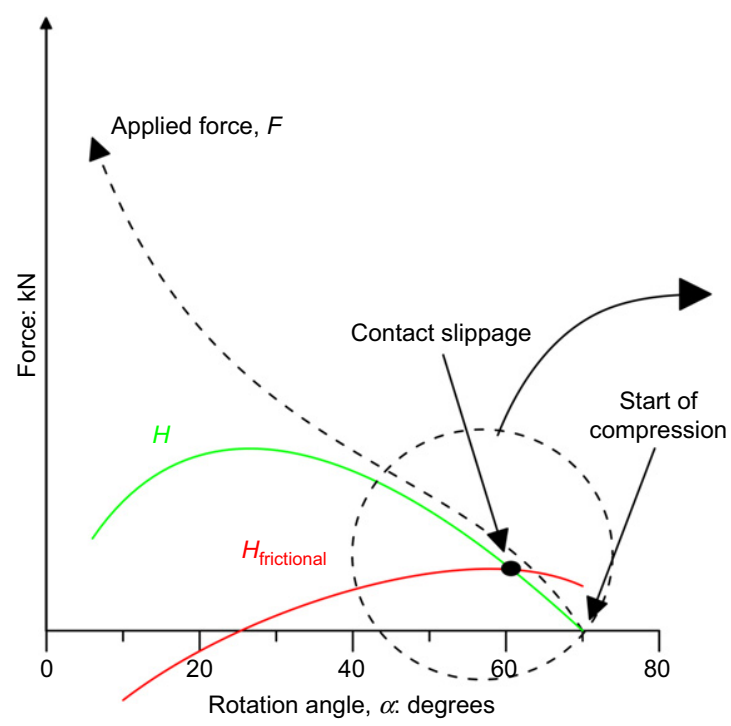

(a)

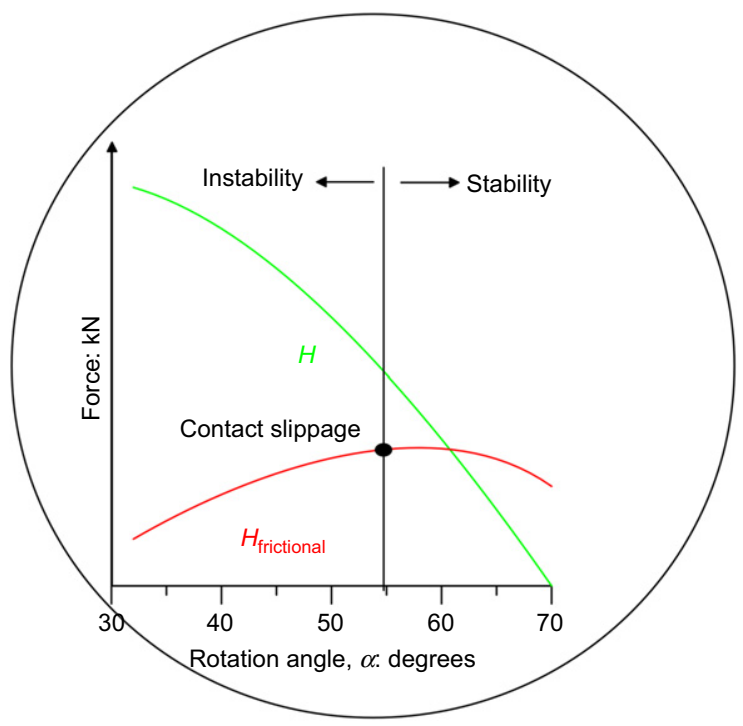

(b)

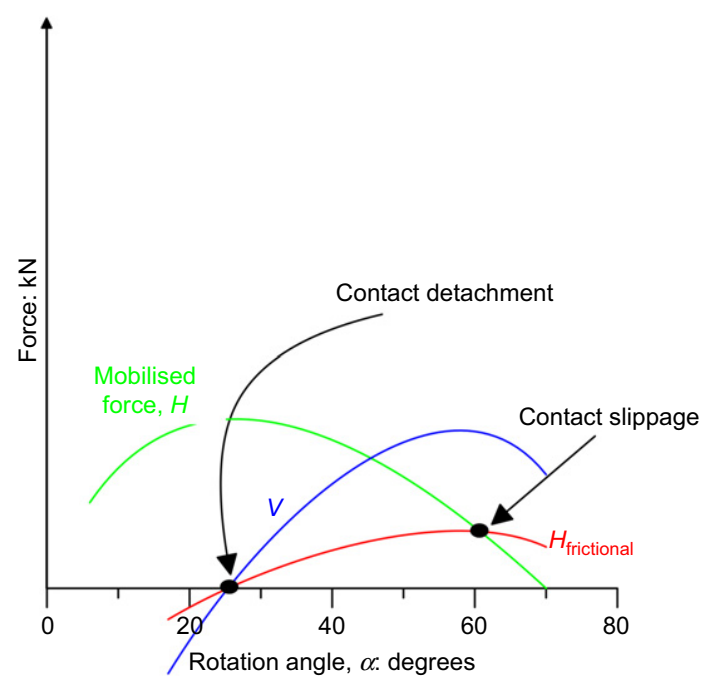

(c)
Contact slippage:

- Stability: $H_{\text {frictional }}>H$

- Instability: $H_{\text {frictional }}>H$

Contact detachment:

- Stability: $V>0$

- Instability: $V<0$ 
adopted to investigate the nature of inter-particle forces was to compare the response of clay prepared with deionised water (characterised by acidic $\mathrm{pH}$ ), which is the 'ordinary' clay that engineers and researchers generally deal with, with clay prepared with alkaline water. The high $\mathrm{pH}$ in alkaline water 'deactivates' the edge-to-face contacts that are indeed active in the clay prepared with deionised (acidic) water.

Inspection of PSDs has revealed that the smaller pores are associated with particles in non-contact configuration, that is, those interacting only by way of the overlap of the repulsive electrical field generated by the negatively charged faces. On the other hand, larger pores are associated with contact configuration generated by the attraction between the positively charged edge and the negatively charged face of the clay particle.

In addition, reversible behaviour appears to be mainly associated with 'elastic' interaction of particles in contact configuration associated with the overlap of the repulsive electrical field. On the other hand, the plastic response appears to be associated with the loss of edge-to-face contacts.

Finally, an embryonic 1D DEM model was developed to show the potential of the micromechanical conceptual model to be implemented into a DEM model. It was assessed qualitatively against the experimental response of clay mixed with pore fluids having different dielectric permittivity values (air, acetone and water).

\section{APPENDIX}

\section{Electric potential}

The electric potential generated by the clay particle surface charge is usually assessed by means of the double layer model. It assumes that the free ions dispersed in a fluid suspension distribute in two layers when in proximity of a charged particle: the Stern layer and the diffuse layer.

This appendix demonstrates that the electrical potential can be computed by neglecting the presence of the free ions in the diffuse layer for the case of a small electrolyte concentration. In other words, the electric potential can be calculated by using the traditional Coulomb's law without invoking the double layer model. The advantage of computing the electric potential by neglecting the diffuse layer allows the electric potential to be estimated also for the case where the pore fluid is non-polar, as is the case for air.

The Poisson's equation (equation (3)) gives the spatial evolution of the electric potential $\phi$ generated by electric charges with density $\rho_{\mathrm{m}}$ in a medium having dielectric permittivity $\varepsilon$

Poisson's equation

$$
\nabla(-\nabla \phi)=-\nabla^{2} \phi=\frac{\rho_{\mathrm{m}}}{\varepsilon}
$$

Two cases can be considered

(a) zero electric charge distribution in the medium; that is, no diffuse layer $(\rho=0)$

(b) electric charge distribution that follows a Boltzmann's distribution (diffuse layer).

If the charge density $\rho$ is equal to zero, Poisson's equation turns into Laplace's equation (equation (4)).

Laplace's equation

$$
\nabla^{2} \phi=0
$$

Solutions of the Laplace's equation can be inferred directly from the definition of the electric potential (equation (5)), which involves little computational effort

$$
\phi(z)=\frac{1}{4 \pi \varepsilon} \frac{\sigma(x)}{|x-z|} d x
$$

where $\sigma$ is the charge distribution on the surface, $d$ is the distance from the surface and $x$ is the spatial coordinate on the surface.
For the case of an electric field generated by a disk having radius $a$ with a uniform surface charge distribution $\sigma_{\mathrm{s}}$ in a free-electrolyte medium having dielectric permittivity $\varepsilon$, the electric potential $\phi(z)$ is can be derived from integration of equation (5)

$$
\phi(z)=\frac{\sigma_{\mathrm{s}}}{2 \varepsilon}\left[\sqrt{\left(a^{2}+z^{2}\right)}-|z|\right]
$$

On the other hand, when the medium is a polar fluid, for example water, and electrolytes are dissolved in it, a distribution of cations and anions is generated. The charge distribution $\rho$ within the medium cannot be considered to be nil. In order to solve the Poisson's equation (equation (3)) it is necessary to assume a distribution of ions in the medium (double layer model) (Gouy, 1910; Chapman, 1913; Stern, 1924; Derjaguin \& Landau, 1941; Verwey et al., 1948; Van Olphen, 1977; Mitchell \& Soga, 2005). According to the Gouy-Chapman theory (Gouy, 1910; Chapman, 1913) a simplified solution for the electric potential, $\phi$, between two plates can be expressed as follows

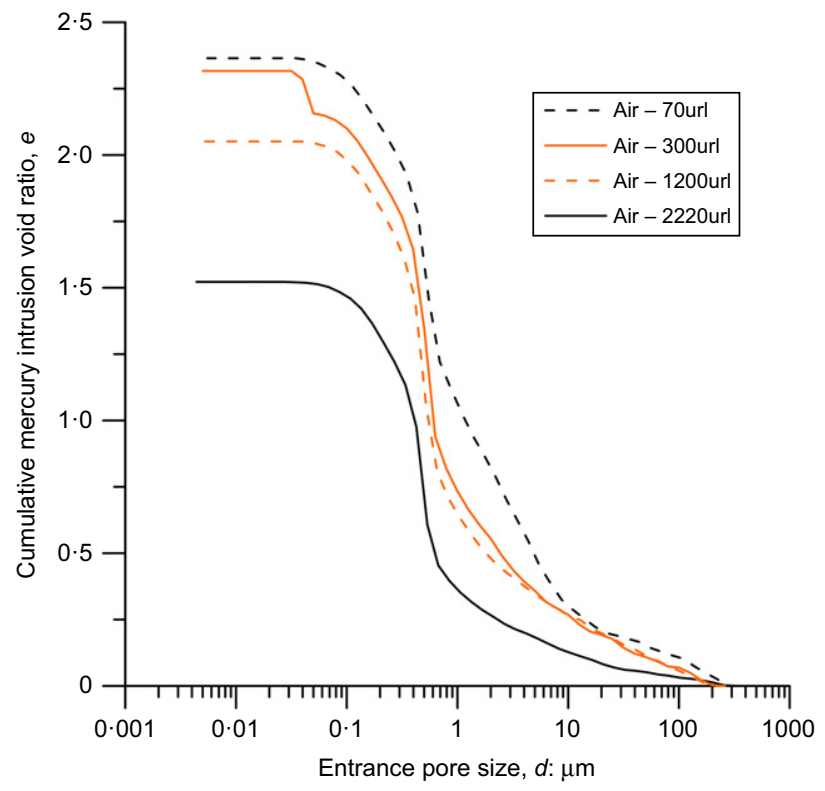

(a)

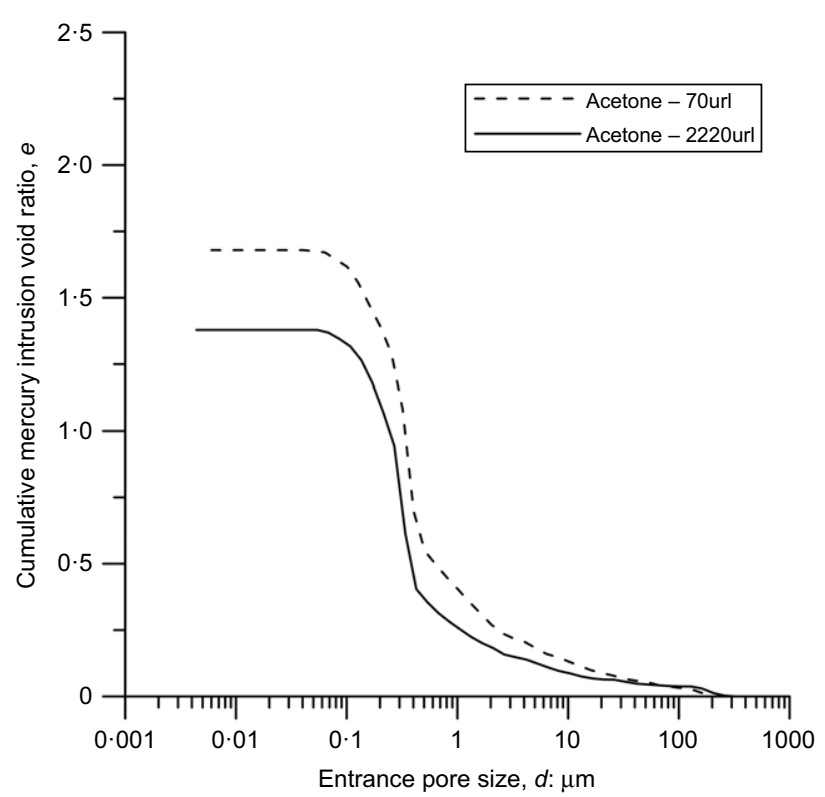

(b)

Fig. 23. Cumulative intrusion void ratio of curves plotted in Fig. 8: (a) air; (b) acetone 


$$
\phi(d) \sim 2\left(\frac{4 k T \gamma_{0}}{v e}\right) \exp (-K z)
$$

where $1 / K$ is defined as the centre of gravity of the diffuse layer and $\gamma_{0}$ is given by

$$
\gamma_{0}=\frac{\exp \left(v e \phi_{0} / 2 k T\right)-1}{\exp \left(v e \phi_{0} / 2 k T\right)+1}
$$

where $k$ is the Boltzmann constant $\left(1.38 \times 10^{-23} \mathrm{~J} / \mathrm{K}\right) ; T(\mathrm{~K})$ is the temperature; $v$ is the ionic valence; $e$ is the electronic charge $\left(1.602 \times 10^{-19} \mathrm{C}\right) ; \phi_{0}$ is the electric potential at the surface; and $K$ is the double layer thickness.

Gouy (1910) and Chapman (1913) presented a simplified solution for the electric potential in this case. Fig. 21(a) shows the electric potential calculated by such a solution for the case of a very small amount of electrolyte in water (sodium chloride with molar concentrations of $10^{-3}, 10^{-4}$ and $10^{-5} \mathrm{M}$ ). The solution using equation (5) (Coulomb's law) is also shown in the same figure. It can be observed that the difference between Gouy-Chapman's solution

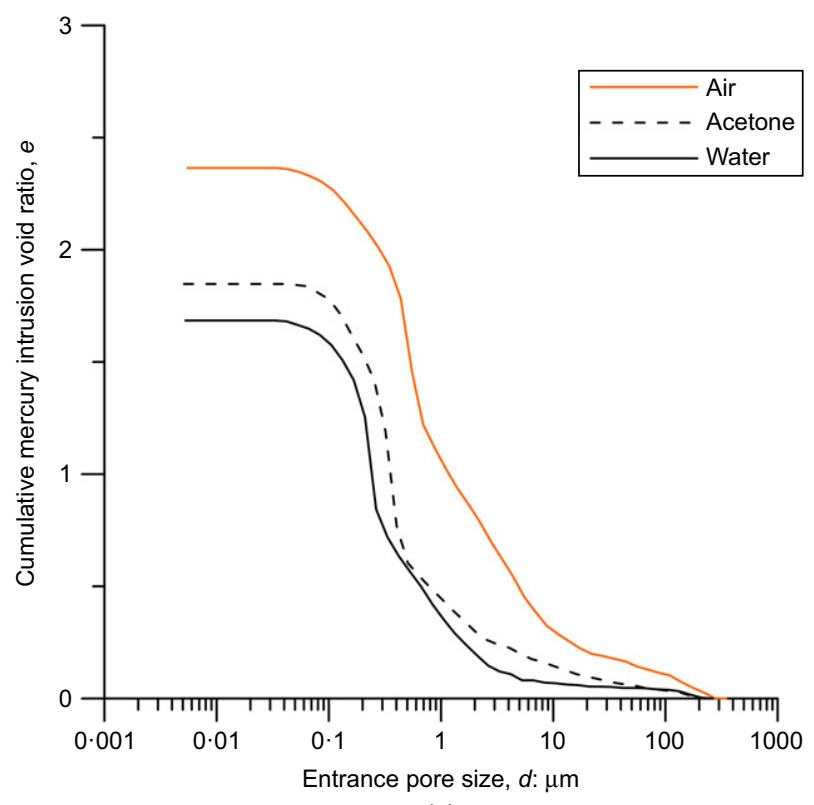

(a)

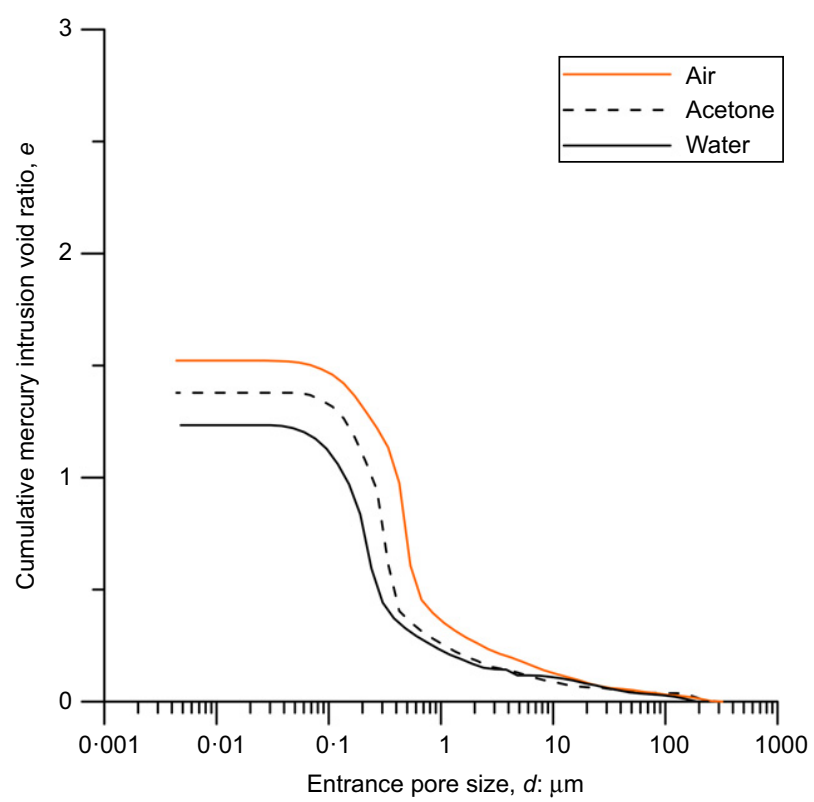

(b)

Fig. 24. Cumulative intrusion void ratio of curves plotted in Fig. 9: (a) consolidation stress $70 \mathrm{kPa}$; (b) consolidation stress $2220 \mathrm{kPa}$ and Coulomb's equation becomes negligible at small electrolyte concentration $\left(\leq 10^{-5} \mathrm{M}\right)$; in other words, Gouy-Chapman's equation tends to reduce to the solution given by equation (5) for the case of no electrical charges in the medium. This electrical conductivity of the pore fluid in the experiments presented in this paper was measured by means of the electric conductivity. The electric conductivity of the pore water was always below $10 \mu \mathrm{S} / \mathrm{cm}$, which corresponds to an equivalent sodium chloride concentration smaller than $2 \times 10^{-5} \mathrm{M}$

The advantage of using Coulomb's equation is that the electric potential can be calculated consistently for the three pore fluids examined in this paper (air, acetone and water). The electrical potentials for these three cases are shown in Fig. 21(b).

\section{Contact mechanism}

In order to understand the mechanisms involved in the disengagement of the contact, the evolution of the constraint reaction forces at the contact was investigated analytically. The horizontal reaction force, $H$, and vertical reaction force, $V$, depend on the

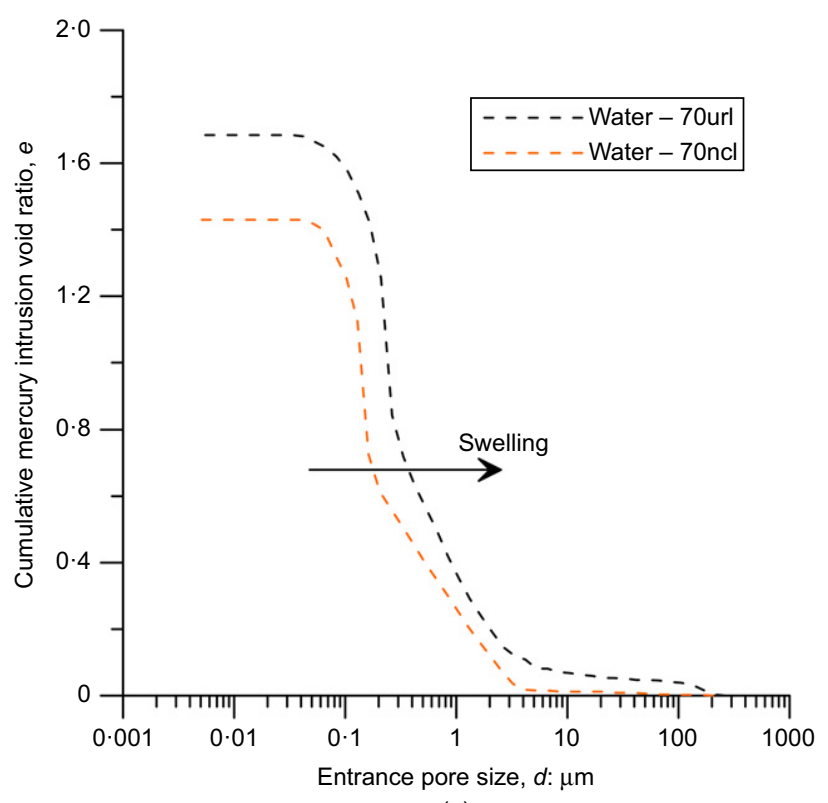

(a)

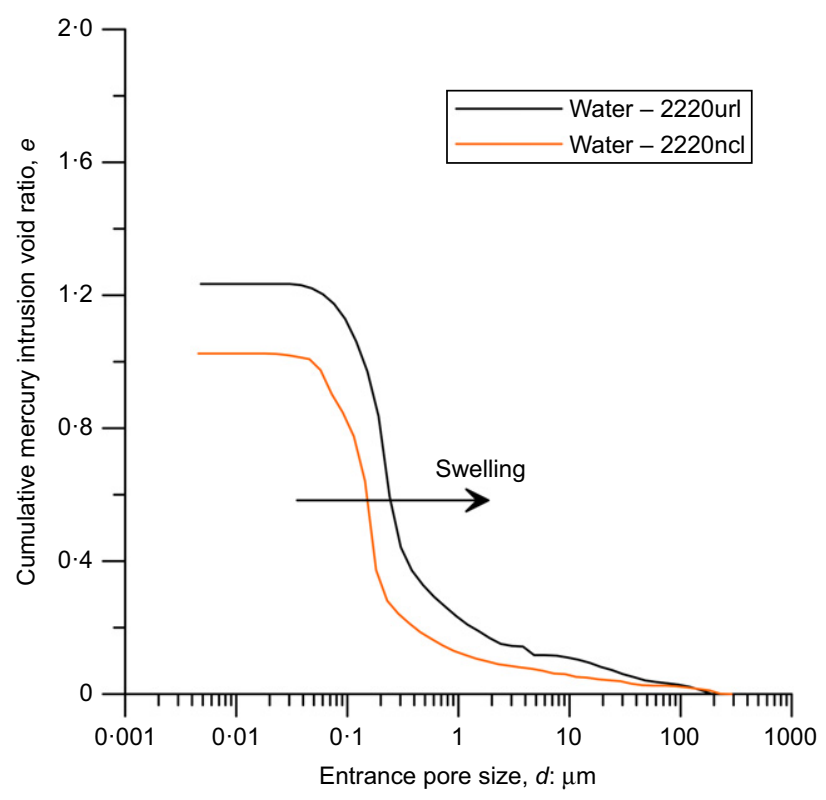

(b)

Fig. 25. Cumulative intrusion void ratio of curves plotted in Fig. 12: (a) consolidation stress $70 \mathrm{kPa}$; (b) consolidation stress $2220 \mathrm{kPa}$ 


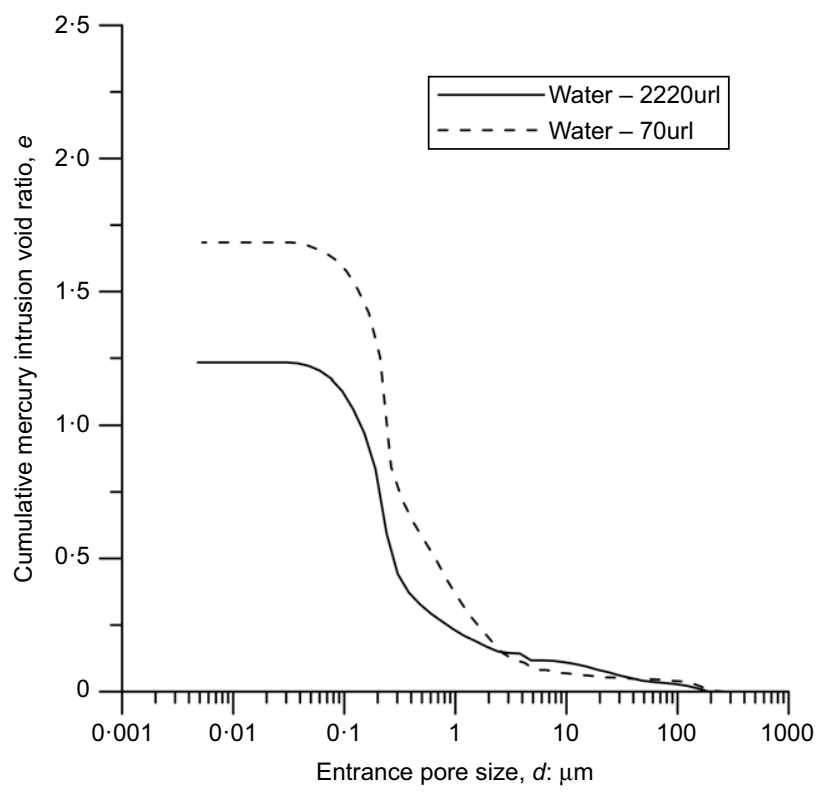

(a)

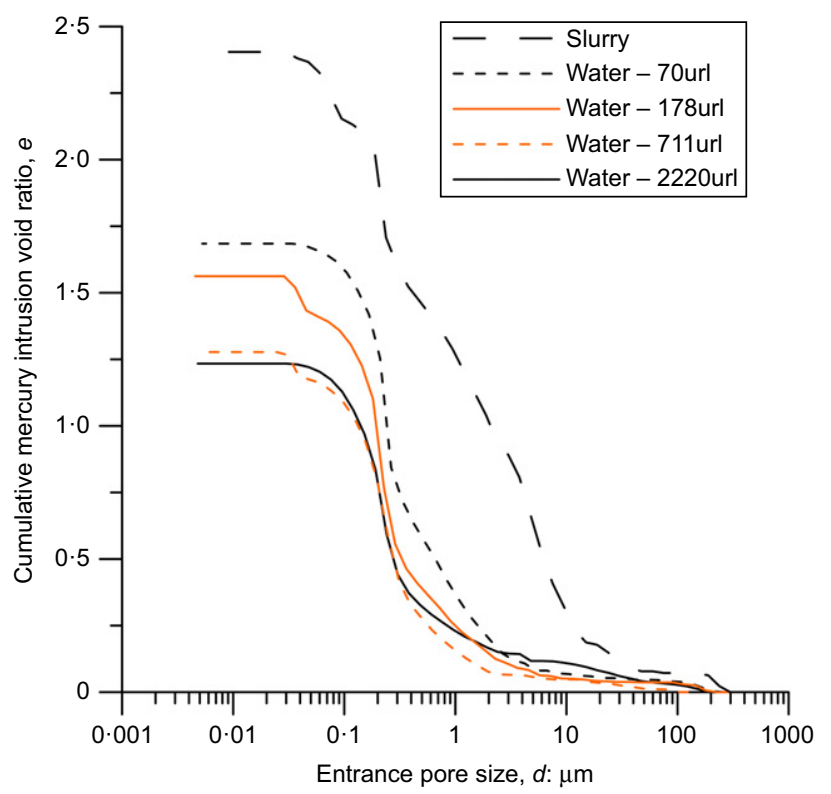

(b)

Fig. 26. Cumulative intrusion void ratio of curves plotted in Fig. 13: (a) consolidation stress $70 \mathrm{kPa}$ and $2220 \mathrm{kPa}$; (b) consolidation stress 70, 178, 711, $2220 \mathrm{kPa}$ and slurry

applied force, $F$, the stiffness of the springs and the tilt angle, $\alpha$, formed by the two particles (Fig. 16).

The vertical reaction, $V$, is computed as the sum of applied force, $F$, the vertical component of the spring reaction, $V_{\text {spring, and the }}$ mutual attraction existing between the edge and the face, acting directly at the contact, $V_{\mathrm{EF}}$ (equation (9)). The horizontal reaction, $H$, is balanced by the horizontal component of the spring reaction,

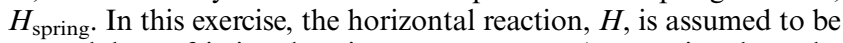
capped by a frictional resistance, $H_{\text {frictional }}$ (proportional to the reaction vertical force $V$ by way of a frictional coefficient $\mu$ ).

$$
\begin{aligned}
& V=F+V_{\text {spring }}+V_{\mathrm{EF}} \\
& H=H_{\text {spring }} \\
& H_{\text {frictional }}=V \mu
\end{aligned}
$$

When the applied external force $F$ is equal to zero, the reaction force $V$ is not equal to zero because of the attraction force $V_{\mathrm{EF}}$ between the two particles.
As long as the contact exists, the constraint at the contact can be thought of as a hinged, fixed bearing (Fig. 16(a)). In Fig. 22(a), the evolution of the reaction forces involved in the contact mechanism against the inter-particle rotation angle, $\alpha$, is reported. For a given starting tilt angle $\left(\alpha=70^{\circ}\right.$ in this exercise), increasing the applied force $F$ causes the particle to tilt and the inter-particle distance to reduce (dashed line in Fig. 22(a)). The rotation of the particles causes $(a)$ the vertical reaction $V$ to reduce and $(b)$ the horizontal reaction force $H$ to increase.

This exercise is seeking to highlight two distinct mechanisms: frictional sliding and detachment.

(a) The particle contact was assumed to be perfectly plastic in the horizontal direction. Slippage occurs when the mobilised horizontal force $H$ becomes greater than the frictional resistance $H_{\text {frictional }}$ (contact slippage in Fig. 22(b)).

(b) The detachment mechanism depends on the mobilised vertical reaction $V$. As the constraint at the contact is unilateral, when the vertical force becomes equal to zero, the particles move apart. In this exercise, the detachment mechanism seems to be more unlikely because it appears to occur always at tilt angles smaller than the one associated with contact slippage (Fig. 22(c)).

\section{Cumulative intrusion void ratio}

The cumulative intrusion void ratios of the PSD curves shown in the paper are reported in Figs 23-26.

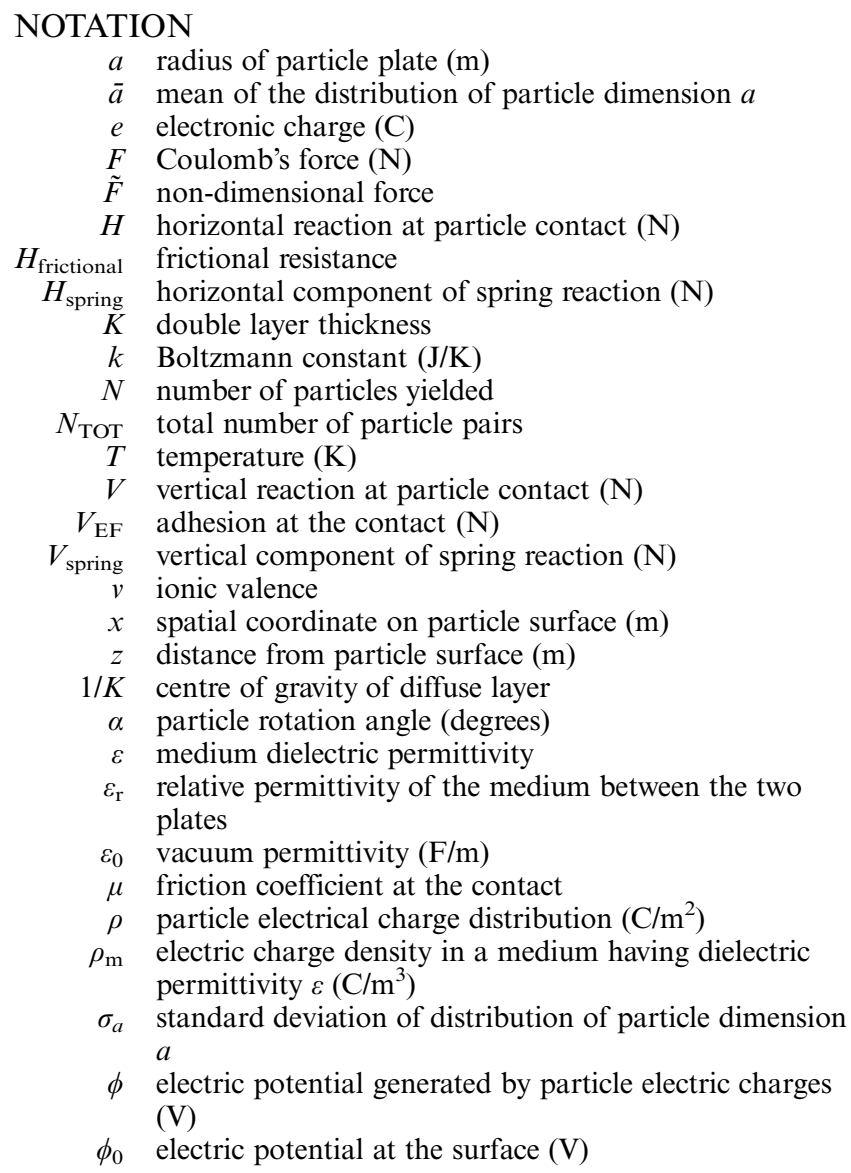

\section{REFERENCES}

Andò, E., Hall, S. A., Viggiani, G., Desrues, J. \& Bésuelle, P. (2012a). Experimental micromechanics: grain-scale observation of sand deformation. Géotechnique Lett. 2, No. 3, 107-112, http://dx.doi.org/10.1680/geolett.12.00027.

Andò, E., Hall, S. A., Viggiani, G., Desrues, J. \& Bésuelle, P. (2012b). Grain-scale experimental investigation of localised 
deformation in sand: a discrete particle tracking approach. Acta Geotechnica 7, No. 1, 1-13.

Bolt, G. (1956). Physico-chemical analysis of the compressibility of pure clays. Géotechnique 6, No. 2, 86-93, http://dx.doi.org/ 10.1680/geot.1956.6.2.86.

Brady, N. C. \& Weil, R. R. (1996). The nature and properties of soils. Upper Saddle River, NJ, USA: Prentice-Hall Inc.

Braggs, B., Fornasiero, D., Ralston, J. \& Smart, R. S. (1994). The effect of surface modification by an organosilane on the electrochemical properties of kaolinite. Clays Clay Miner. 42, No. 2, 123-136.

BSI (1990). BS 1377:1990: Methods of test for soils for civil engineering purposes. London, UK: BSI.

Carroll, S. A. \& Walther, J. V. (1990). Kaolinite dissolution at 25, 60 and $80^{\circ}$ C. Am. J. Sci. 290, No. 7, 797-810.

Carty, W. (1999). The colloidal nature of kaolinite. Am. Ceramic Soc. Bull. 78, No. 8, 72-76.

Chapman, D. L. (1913). LI. A contribution to the theory of electrocapillarity. London, Edinburgh, Dublin Phil. Mag. J. Sci. 25, No. 148, 475-481.

Chen, J., Anandarajah, A. \& Inyang, H. (2000). Pore fluid properties and compressibility of kaolinite. J. Geotech. Geoenviron. Engng 126, No. 9, 798-807.

Delage, P. (2010). A microstructure approach to the sensitivity and compressibility of some Eastern Canada sensitive clays. Géotechnique 60, No. 5, 353-368, http://dx.doi.org/10. 1680/geot.2010.60.5.353.

Delage, P. \& Lefebvre, G. (1984). Study of the structure of a sensitive Champlain clay and of its evolution during consolidation. Can. Geotech. J. 21, No. 1, 21-35.

Derjaguin, B. \& Landau, L. (1941). Theory of the stability of strongly charged lyophobic sols and of the adhesion of strongly charged particles in solutions of electrolytes. Acta Physicochimica URSS 14, No. 6, 633-662.

Di Maio, C. (1996). Exposure of bentonite to salt solution: osmotic and mechanical effects. Géotechnique 46, No. 4, 695-707, http://dx.doi.org/10.1680/geot.1996.46.4.695.

Di Maio, C. \& Fenelli, G. (1994). Residual strength of kaolin and bentonite: the influence of their constituent pore fluid. Géotechnique 44, No. 2, 217-226, http://dx.doi.org/10. 1680/geot.1994.44.2.217.

Gajo, A. \& Loret, B. (2007). The mechanics of active clays circulated by salts, acids and bases. J. Mech. Phys. Solids 55, No. 8, 1762-1801.

Ganor, J., Mogollón, J. L. \& Lasaga, A. C. (1995). The effect of $\mathrm{pH}$ on kaolinite dissolution rates and on activation energy. Geochimica et Cosmochimica Acta 59, No. 6, 1037-1052.

Gouy, G. (1910). Constitution of the electric charge at the surface of an electrolyte. J. Physics 9, No. 4, 457-467.

Gupta, V. \& Miller, J. D. (2010). Surface force measurements at the basal planes of ordered kaolinite particles. J. Colloid Interface Sci. 344, No. 2, 362-371.

Gupta, V., Hampton, M. A., Stokes, J. R., Nguyen, A. V. \& Miller, J. D. (2011). Particle interactions in kaolinite suspensions and corresponding aggregate structures. J. Colloid Interface Sci. 359, No. 1, 95-103.

Hattab, M., Bouziri-Adrouche, S. \& Fleureau, J. M. (2010). Évolution de la microtexture d'une matrice kaolinitique sur chemin triaxial axisymétrique. Can. Geotech. J. 47, No. 1, 34-48 (in French).

Huertas, F. J., Chou, L. \& Wollast, R. (1998). Mechanism of kaolinite dissolution at room temperature and pressure: Part 1. Surface speciation. Geochimica et Cosmochimica Acta 62, No. 3, 417-431.

Kretzschmar, R., Holthoff, H. \& Sticher, H. (1998). Influence of $\mathrm{pH}$ and humic acid on coagulation kinetics of kaolinite: a dynamic light scattering study. J. Colloid Interface Sci. 202, No. 1, 95-103.
Madsen, F. T. \& Müller-Vonmoos, M. (1989). The swelling behaviour of clays. Appl. Clay Sci. 4, No. 2, 143-156.

Mitchell, J. \& Soga, K. (2005). Fundamentals of soil behavior. Hoboken, NJ, USA: John Wiley and Sons Inc.

Oda, M. \& Kazama, H. (1998). Microstructure of shear bands and its relation to the mechanisms of dilatancy and failure of dense granular soils. Géotechnique 48, No. 4, 465-481, http://dx.doi. org/10.1680/geot.1998.48.4.465.

Oda, M., Kazama, H. \& Konishi, J. (1998). Effects of induced anisotropy on the development of shear bands in granular materials. Mech. Mater. 28, No. 4, 103-111.

O'Sullivan, C. (2011). Particulate discrete element modelling. Hoboken, NJ, USA: Taylor \& Francis.

Pedrotti, M. (2016). An experimental investigation on the micromechanics of non-active clays in saturated and partially-saturated states. $\mathrm{PhD}$ thesis, University of Strathclyde, Glasgow, UK.

Rand, B. \& Melton, I. E. (1977). Particle interactions in aqueous kaolinite suspensions: I. Effect of $\mathrm{pH}$ and electrolyte upon the mode of particle interaction in homoionic sodium kaolinite suspensions. J. Colloid Interface Sci. 60, No. 2, 308-320.

Roscoe, K. H. \& Burland, J. (1968). On the generalized stress-strain behaviour of wet clay. In Engineering plasticity (eds J. Heyman and F. A. Leckie), pp. 535-609. Cambridge, UK: Cambridge University Press.

Roscoe, K., Schofield, A. \& Thurairajah, A. (1963). Yielding of clays in states wetter than critical. Géotechnique 13, No. 3, 211-240, http://dx.doi.org/10.1680/geot.1963.13.3.211.

Sachan, A. \& Penumadu, D. (2007). Effect of microfabric on shear behavior of kaolin clay. J. Geotech. Geoenviron. Engng 133, No. 3, 306-318.

Schofield, R. \& Samson, H. (1954). Flocculation of kaolinite due to the attraction of oppositely charged crystal faces. Discussions Faraday Soc. 18, 135-145.

Sloane, R. L. \& Kell, T. (1966). The fabric of mechanically compacted kaolin. Clays Clay Miner. 14, No. 1, 289-295.

Sposito, G. (1984). The surface chemistry of soils. New York, NY, USA: Oxford University Press.

Sridharan, A. \& Venkatappa Rao, G. (1973). Mechanisms controlling volume change of saturated clays and the role of the effective stress concept. Géotechnique 23, No. 3, 359-382, http://dx.doi. org/10.1680/geot.1973.23.3.359.

Stern, O. (1924). The theory of the electrolytic double-layer. Zeit. Elektrochem. 30, 508-516.

Tarantino, A. \& De Col, E. (2008). Compaction behaviour of clay. Géotechnique 58, 199-213, http://dx.doi.org/10.1680/geot.2008. 58.3.199.

Van Olphen, H. (1977). Clay colloid chemistry: for clay technologists, geologists and soil scientists. New York, NY, USA: John Wiley.

Verwey, E. J. W., Overbeek, J. T. G. \& Van Nes, K. (1948). Theory of the stability of lyophobic colloids: the interaction of sol particles having an electric double layer. New York, NY, USA: Elsevier.

Wahid, A. S., Gajo, A. \& Di Maggio, R. (2011a). Chemo-mechanical effects in kaolinite. Part 1: prepared samples. Géotechnique 61, No. 6, 439-447, http://dx.doi. org/10.1680/geot.8.P.067.

Wahid, A. S., Gajo, A. \& Di Maggio, R. (2011b). Chemo-mechanical effects in kaolinite. Part 2: exposed samples and chemical and phase analyses. Géotechnique 61, No. 6, 449-457, http://dx.doi.org/10.1680/geot.8.P.068.

Wang, Y. H. \& Siu, W. K. (2006). Structure characteristics and mechanical properties of kaolinite soils. I. Surface charges and structural characterizations. Can. Geotech. J. 43, No. 6, $587-600$.

Zhao, B., Wang, J., Coop, M. R., Viggiani, C. \& Jiang, M. (2015). An investigation of single sand particle fracture using X-ray micro-tomography. Géotechnique 65, No. 8, 625-641, http://dx. doi.org/10.1680/geot.4.P.157. 